\title{
UNIFORM STABILIZATION \\ OF THE HIGHER-DIMENSIONAL SYSTEM \\ OF THERMOELASTICITY \\ WITH A NONLINEAR BOUNDARY FEEDBACK
}

\author{
BY \\ WEI-JIU LIU (Department of Mathematics and Statistics, Dalhousie University, Halifax, Nova \\ Scotia, Canada) \\ AND \\ ENRIQUE ZUAZUA (Departamento de Matemática Aplicada, Universidad Complutense de \\ Madrid, Spain)
}

\begin{abstract}
Using multiplier techniques and Lyapunov methods, we derive explicit decay rates for the energy in the higher-dimensional system of thermoelasticity with a nonlinear velocity feedback on part of the boundary of a thermoelastic body, which is clamped along the rest of its boundary.
\end{abstract}

1. Introduction. Let $\Omega$ be a bounded domain in $\mathbf{R}^{n}$ with smooth boundary $\Gamma=\partial \Omega$ of class $C^{2}$, and consider an $n$-dimensional linear, homogeneous, isotropic, and thermoelastic body occupying $\Omega$ in its non-deformed state. For a material point with configuration $x=\left(x_{1}, \ldots, x_{n}\right)$ at time $t$, let $u(x, t)=\left(u_{1}(x, t), \ldots, u_{n}(x, t)\right)$ and $\theta(x, t)$ denote the displacement and temperature deviation, respectively, from the natural state of the reference configuration. Then, in the absence of external forces and heat sources, $u$ and $\theta$ satisfy the system of equations of thermoelasticity

$$
\begin{cases}u^{\prime \prime}-\mu \Delta u-(\lambda+\mu) \nabla \operatorname{div} u+\alpha \nabla \theta=0 & \text { in } \Omega \times(0, \infty), \\ \theta^{\prime}-\Delta \theta+\beta \operatorname{div} u^{\prime}=0 & \text { in } \Omega \times(0, \infty), \\ u(0)=u^{0}, u^{\prime}(0)=u^{1}, \theta(0)=\theta^{0} & \text { in } \Omega,\end{cases}
$$

where $\alpha, \beta>0$ are the coupling parameters and $\lambda, \mu$ are Lamé's constants satisfying

$$
\mu>0, \quad n \lambda+(n+1) \mu>0 .
$$

Received November 24, 1998.

2000 Mathematics Subject Classification. Primary 35B35, 74B05.

Key words and phrases. Thermoelasticity, uniform boundary stabilization, Lyapunov methods.

The work of the first author was supported in part by the Killam Postdoctoral Fellowship and done in part while the author was with UC San Diego.

The work of the second author was supported by grant PB96-0663 of the DGES (Spain).

E-mail address: weiliu@mathstat.dal.ca

E-mail address: zuazua@sunma4.mat.ucm.es 
By ' we denote the derivative with respect to the time variable. $\Delta, \nabla$, div denote the Laplace, gradient, and divergence operators in the space variables respectively. $u(0), u^{\prime}(0)$ and $\theta(0)$ denote the functions $x \rightarrow u(x, 0), x \rightarrow u^{\prime}(x, 0)$ and $x \rightarrow \theta(x, 0)$, respectively. For the derivation of (1.1), we refer to [24] and [44].

Note that condition (1.2) is weaker than the following usual condition on the Lamé coefficients (see [44] and [13, p. 414])

$$
n \lambda+2 \mu>0 .
$$

Extensive work has been done on the problem of stabilization for system (1.1) (see $[5,9,7,12,17,20,27,33,37,40,42,43,45,46,47,48])$. We give here a brief description of the existing literature. For a detailed survey, we refer to $[37,38]$.

The thermoelastic energy of (1.1) can be defined as

$$
\begin{aligned}
E(u, \theta, t)= & \frac{1}{2} \int_{\Omega}\left[\left|u^{\prime}(x, t)\right|^{2}+\mu|\nabla u(x, t)|^{2}\right. \\
& \left.+(\lambda+\mu)|\operatorname{div} u(x, t)|^{2}+\frac{\alpha}{\beta}|\theta(x, t)|^{2}\right] d x .
\end{aligned}
$$

Here we have used the notation

$$
|\nabla u(x, t)|^{2}=\sum_{i, j=1}^{n}\left|\frac{\partial u_{i}}{\partial x_{j}}\right|^{2} .
$$

Under the Dirichlet-Dirichlet boundary conditions

$$
u=0, \theta=0 \quad \text { on } \Gamma \times(0, \theta)
$$

it is easy to verify that

$$
E^{\prime}(u, \theta, t)=-\frac{\alpha}{\beta} \int_{\Omega}|\nabla \theta(x, t)|^{2} d x .
$$

Therefore, the energy $E(u, \theta, t)$ decreases on $(0, \infty)$. Furthermore, in the case of one space dimension, it has been shown (see $[9,17,20,40]$ ) that the energy $E(u, \theta, t)$ of system (1.1) associated with various boundary conditions decays to zero exponentially. In the case of multi-space dimension, Dafermos in his pioneering work [12] showed that, generically with respect to the domain, the energy of every solution of (1.1) and (1.6) tends to zero as $t \rightarrow \infty$. However, he also pointed out that, when $\Omega$ is a ball, non-decaying solutions do exist. More recently, Lebeau and Zuazua [33] proved that the decay rate is never uniform when $\Omega$ is convex. Thus, in order to ensure the uniform decay rate for such convex domains, additional damping mechanisms are necessary. In this aspect, the first author (see [37]) introduced a linear boundary velocity feedback acting on the elastic component of the system and established the uniform decay rate.

The purpose of this paper is to introduce a nonlinear boundary feedback which allows us to test the robustness of the damping mechanisms. Under the classical polynomial growth assumption on the nonlinear boundary feedback near the origin, by using multiplier techniques and Lyapunov methods, we show that the energy in the multidimensional system of thermoelasticity decays to zero at an exponential or polynomial rate. 
Further, even if the nonlinearity does degenerate at the origin faster than any polynomial, we show that the decay rate is governed by a dissipative ordinary differential equation. This allows us to show, in particular, that if the nonlinearity degenerates at the origin exponentially, then we obtain a logarithmic decay rate. To do that, we proceed as in [41] where the simpler case of the wave equation with internal damping is addressed. This type of result was obtained earlier by Lasiecka et al [32] for the wave equation with nonlinear boundary feedback. However, our approach, even if it uses some ingredients as in [32] (for instance, Jensen's inequality), relies essentially on the generalized Young's inequality and it is simpler. This allows us to get more explicit expressions for the nonlinearity entering in the differential inequality governing the decay of the energy.

The rest of this paper is organized as follows. In Section 2, we present our main results. In Section 3, we prove that the system of thermoelasticity with a nonlinear boundary feedback is well-posed by using the theory of nonlinear semigroups. Then, borrowing Lyapunov methods and multiplier techniques, we prove our main results in Section 4. Finally, in Section 5, we briefly discuss some special case and pose an open problem.

2. Main results. Let $\Omega$ be a bounded domain in $\mathbf{R}^{n}$ with smooth boundary $\Gamma=\partial \Omega$ of class $C^{2}$. Set

$$
\begin{aligned}
& \Gamma_{1}=\{x \in \Gamma: m(x) \cdot \nu(x) \leq 0\}, \\
& \Gamma_{2}=\{x \in \Gamma: m(x) \cdot \nu(x)>0\},
\end{aligned}
$$

where

$$
m(x)=x-x^{0}=\left(x_{1}-x_{1}^{0}, \ldots, x_{n}-x_{n}^{0}\right)
$$

for some $x^{0} \in \mathbf{R}^{n}, \nu=\left(\nu_{1}, \ldots, \nu_{n}\right)$ denotes the unit normal on $\Gamma$ directed towards the exterior of $\Omega$ and

$$
m \cdot \nu=m(x) \cdot \nu(x)=\sum_{i=1}^{n}\left(x_{i}-x_{i}^{0}\right) \nu_{i}
$$

$\Gamma_{1}$ is assumed either to be empty or to have a nonempty interior relative to $\Gamma$.

In what follows, $H^{s}(\Omega)$ denotes the usual Sobolev space (see [1]) for any $s \in \mathbf{R}$. For $s \geq 0, H_{0}^{s}(\Omega)$ denotes the completion of $C_{0}^{\infty}(\Omega)$ in $H^{s}(\Omega)$, where $C_{0}^{\infty}(\Omega)$ denotes the space of all infinitely differentiable functions on $\Omega$ with compact support in $\Omega$. Let $X$ be a Banach space. We denote by $C^{k}([0, T] ; X)$ the space of all $k$ times continuously differentiable functions defined on $[0, T]$ with values in $X$, and write $C([0, T] ; X)$ for $C^{0}([0, T] ; X)$.

We further introduce other function spaces as follows:

$$
\begin{aligned}
H_{\Gamma_{1}}^{1}(\Omega) & =\left\{u \in H^{1}(\Omega): u=0 \text { on } \Gamma_{1}\right\}, \\
\mathcal{H} & =\left(H_{\Gamma_{1}}^{1}(\Omega)\right)^{n} \times\left(L^{2}(\Omega)\right)^{n} \times L^{2}(\Omega) .
\end{aligned}
$$


We consider the thermoclastic system with a nonlinear boundary feedback

$$
\begin{cases}u^{\prime \prime}-\mu \Delta u-(\lambda+\mu) \nabla \operatorname{div} u+\alpha \nabla \theta=0 & \text { in } \Omega \times(0, \infty), \\ \theta^{\prime}-\Delta \theta+\beta \operatorname{div} u^{\prime}=0 & \text { in } \Omega \times(0, \infty), \\ \theta=0 & \text { on } \Gamma \times(0, \infty), \\ u=0 & \text { on } \Gamma_{1} \times(0, \infty), \\ \mu \frac{\partial u}{\partial \nu}+(\lambda+\mu) \operatorname{div}(u) \nu & \\ \quad+a m \cdot \nu u+m \cdot \nu g\left(u^{\prime}\right)=0 & \text { on } \Gamma_{2} \times(0, \infty), \\ u(0)=u^{0}, u^{\prime}(0)=u^{1}, \theta(0)=\theta^{0} & \text { in } \Omega,\end{cases}
$$

where $a=a(x)$ is a given nonnegative function on $\Gamma_{2}$ with

$$
a(x) \in C^{1}\left(\Gamma_{2}\right),
$$

and $g(u)=\left(g_{1}\left(u_{1}, \ldots, u_{n}\right), \ldots, g_{n}\left(u_{1}, \ldots, u_{n}\right)\right) \in\left(C\left(\mathbf{R}^{n}\right)\right)^{n}$ is a given vector function. Similar nonlinear boundary feedbacks were introduced for the wave equation (see $[11,31$, $32,50,51])$ and the equations of elasticity (sec $[28,29])$.

The elastic Lamé operator $\mu \Delta u-(\lambda+\mu) \nabla \operatorname{div} u$ may be written in divergence form $\operatorname{div}\left[\sigma_{i j}(u)\right]$, where the stress tensor $\sigma_{i j}(u)$ is given by

$$
\sigma_{i j}(u)=2 \mu \varepsilon_{i j}(u)+\lambda \delta_{i j} \sum_{k=1}^{n} \varepsilon_{k k}(u)
$$

and the linearized strain tensor $\varepsilon_{i j}(u)$ is given by

$$
\varepsilon_{i j}(u)=\frac{1}{2}\left(\frac{\partial u_{i}}{\partial x_{j}}+\frac{\partial u_{j}}{\partial x_{i}}\right) .
$$

The boundary conditions describing the surface forces are given by

$$
\sigma_{i j}(u) \nu_{j}+a m \cdot \nu u_{i}=f_{i} .
$$

Since

$$
\mu \frac{\partial u_{i}}{\partial \nu}+(\lambda+\mu) \operatorname{div}(u) \nu_{i}=\sigma_{i j}(u) \nu_{j}+\mu\left(\frac{\partial u_{j}}{\partial x_{j}} \nu_{i}-\frac{\partial u_{j}}{\partial x_{i}} \nu_{j}\right),
$$

the boundary condition in $(2.7)$

$$
\mu \frac{\partial u}{\partial \nu}+(\lambda+\mu) \operatorname{div}(u) \nu+a m \cdot \nu u+m \cdot \nu g\left(u^{\prime}\right)=0
$$

can be easily transferred into (2.11) with

$$
f_{i}(u)=-m \cdot \nu g_{i}\left(u^{\prime}\right)-\mu\left(\frac{\partial u_{j}}{\partial x_{j}} \nu_{i}-\frac{\partial u_{j}}{\partial x_{i}} \nu_{j}\right) .
$$

Therefore, system (2.7) may be viewed as the system of thermoelasticity subject to a boundary feedback force of the form (2.14). This feedback mechanism is however nonoptimal since, due to the presence of the first-order space derivates, its regularity is not sharp (one cannot guarantee that it belongs to $L^{2}(\partial \Omega \times(0, T))$ for finite energy solutions). Therefore, the question of analyzing the stabilization under the weaker feedback forces of the form

$$
f_{i}(u)=-m \cdot \nu g_{i}\left(u^{\prime}\right)
$$


in which the last term in (2.14) has been dropped, is an interesting open problem. This analysis has been performed in $[3,19]$ in the context of the system of elasticity but, to our knowledge, this issue has not been addressed for the system of thermoelasticity.

Throughout this paper, we assume that

$$
\Gamma_{1} \neq \emptyset \quad \text { or } \quad a(x) \not \equiv 0
$$

We refer to Section 5 for a brief discussion of the case where $\Gamma_{1}=\emptyset$ and $a(x) \equiv 0$. Under assumptions (1.2) and (2.16), one can easily show that the following norm on $\left(H_{\Gamma_{1}}^{1}(\Omega)\right)^{n}$

$$
\begin{aligned}
\|u\|_{\left(H_{\Gamma_{1}}^{1}(\Omega)\right)^{n}}= & \left(\int_{\Omega}\left[\mu|\nabla u|^{2}+(\lambda+\mu)|\operatorname{div}(u)|^{2}\right] d x\right. \\
& \left.+\int_{\Gamma_{2}} a m \cdot \nu|u|^{2} d \Gamma\right)^{1 / 2}
\end{aligned}
$$

is equivalent to the usual one induced by $\left(H^{1}(\Omega)\right)^{n}$ (see [37]). Indeed, it suffices to note the following fact:

$$
\mu|\nabla u|^{2}+(\lambda+\mu)|\operatorname{div} u|^{2} \geq \mu|\nabla u|^{2}, \quad \text { for } \lambda+\mu \geq 0
$$

and for $\lambda+\mu<0$,

$$
\begin{aligned}
\mu|\nabla u|^{2}+(\lambda+\mu)|\operatorname{div} u|^{2} & =\mu \sum_{i, j=1}^{n}\left|\frac{\partial u_{i}}{\partial x_{j}}\right|^{2}+(\lambda+\mu) \sum_{i, j=1}^{n} \frac{\partial u_{i}}{\partial x_{i}} \frac{\partial u_{j}}{\partial x_{j}} \\
& \geq \mu \sum_{i, j=1}^{n}\left|\frac{\partial u_{i}}{\partial x_{j}}\right|^{2}+(\lambda+\mu) n \sum_{i}^{n}\left|\frac{\partial u_{i}}{\partial x_{i}}\right|^{2} \\
& =\mu \sum_{i \neq j}\left|\frac{\partial u_{i}}{\partial x_{j}}\right|^{2}+[n \lambda+(n+1) \mu] \sum_{i}^{n}\left|\frac{\partial u_{i}}{\partial x_{i}}\right|^{2} \\
& \geq \min \{\mu, n \lambda+(n+1) \mu\}|\nabla u|^{2} .
\end{aligned}
$$

In the sequel, we use the following energy norm on $\mathcal{H}$ :

$$
\begin{aligned}
\|(u, v, \theta)\|_{\mathcal{H}}= & \left(\int_{\Omega}\left[\mu|\nabla u|^{2}+(\lambda+\mu)|\operatorname{div}(u)|^{2}+|v|^{2}+\frac{\alpha}{\beta}|\theta|^{2}\right] d x\right. \\
& \left.+\int_{\Gamma_{2}} a m \cdot \nu|u|^{2} d \Gamma\right)^{1 / 2}
\end{aligned}
$$

for $(u, v, \theta) \in \mathcal{H}$, which is equivalent to the usual one induced by $\left(H^{1}(\Omega)\right)^{n} \times\left(L^{2}(\Omega)\right)^{n} \times$ $L^{2}(\Omega)$.

In Section 3, we will prove that system (2.7) generates a nonlinear continuous semigroup $S(t)$ on $\mathcal{H}$. Thus, system (2.7) is well-posed. 
In order to state our main results, we first introduce some constants. In what follows, we denote by $\|\cdot\|$ the norm of $L^{2}(\Omega)$ or $\left(L^{2}(\Omega)\right)^{n}$. Set

$$
\begin{aligned}
R_{0} & =\max _{x \in \bar{\Omega}}|m(x)|=\max _{x \in \bar{\Omega}}\left|\sum_{k=1}^{n}\left(x_{k}-x_{k}^{0}\right)^{2}\right|^{1 / 2}, \\
a_{0} & =\max _{x \in \Gamma_{2}} a(x), \\
K(a) & =\frac{2 a_{0}^{2} R_{0}^{2}}{\mu}+(2-n) a_{0} .
\end{aligned}
$$

Let $\gamma$ be the smallest positive constant such that

$$
\int_{\Gamma_{2}}|u|^{2} d \Gamma \leq \gamma^{2}\|u\|_{\left(H_{\Gamma_{1}}^{1}(\Omega)\right)^{n}}^{2}, \quad \forall u \in\left(H_{\Gamma_{1}}^{1}(\Omega)\right)^{n} .
$$

Let $\lambda_{0}$ and $\lambda_{1}$ be the smallest positive constants, respectively, such that

$$
\|u\| \leq \lambda_{0}\|u\|_{\left(H_{\Gamma_{1}}^{1}(\Omega)\right)^{n}}, \quad \forall u \in\left(H_{\Gamma_{1}}^{1}(\Omega)\right)^{n},
$$

and

$$
\|u\| \leq \lambda_{1}\|\nabla u\|, \quad \forall u \in H_{0}^{1}(\Omega) .
$$

Since the decay rates depend also on the constant appearing in the following technical lemma, we present it before stating our main results. This lemma is helpful for dealing with the case where the potential $a$ is large.

LEMmA 2.1. There exists a constant $k_{1}>0$, independent of $u$, such that the solution $\varphi$ of

$$
\begin{cases}\mu \Delta \varphi+(\lambda+\mu) \nabla \operatorname{div} \varphi=0 & \text { in } \Omega \\ \varphi=u & \text { on } \Gamma\end{cases}
$$

satisfies

$$
\|\varphi\|^{2} \leq k_{1}^{2} \int_{\Gamma}|u|^{2} d \Gamma
$$

and

$$
\int_{\Omega}[\mu \nabla u \cdot \nabla \varphi+(\lambda+\mu) \operatorname{div}(u) \operatorname{div}(\varphi)] d x \geq 0,
$$

for all $u \in\left(H^{1}(\Omega)\right)^{n}$, where

$$
\nabla u \cdot \nabla \varphi=\sum_{i=1}^{n} \nabla u_{i} \cdot \nabla \varphi_{i}
$$

Proof. For any $f \in\left(L^{2}(\Omega)\right)^{n}$, we consider

$$
\begin{cases}\mu \Delta \psi+(\lambda+\mu) \nabla \operatorname{div} \psi=f & \text { in } \Omega, \\ \psi=0 & \text { on } \Gamma .\end{cases}
$$

Multiplying (2.31) by $\varphi$ and integrating over $\Omega$ by parts, we obtain

$$
\int_{\Omega} \varphi \cdot f d x=\int_{\Gamma}\left[\mu \frac{\partial \psi}{\partial \nu}+(\lambda+\mu) \operatorname{div}(\psi) \nu\right] \cdot u d \Gamma .
$$


On the other hand, there exists a constant $c>0$ such that

$$
\left\|\mu \frac{\partial \psi}{\partial \nu}+(\lambda+\mu) \operatorname{div}(\psi) \nu\right\|_{\left(L^{2}(\Gamma)\right)^{n}} \leq c\|f\|, \quad \forall f \in\left(L^{2}(\Omega)\right)^{n} .
$$

To prove this inequality, we let $h=\left(h_{1}, \ldots, h_{n}\right)$ be a vector field in $\left(C^{1}(\bar{\Omega})\right)^{n}$ satisfying

$$
h=\nu \quad \text { on } \Gamma \text {. }
$$

Multiplying (2.31) by $h \cdot \nabla \psi$ and integrating over $\Omega$ by parts, we obtain

$$
\begin{aligned}
\int_{\Omega} \Delta \psi_{i} h_{k} \frac{\partial \psi_{i}}{\partial x_{k}}= & \frac{1}{2} \int_{\Gamma} h \cdot \nu\left|\frac{\partial \psi_{i}}{\partial \nu}\right|^{2} d \Gamma+\frac{1}{2} \int_{\Omega} \operatorname{div}(h)\left|\nabla \psi_{i}\right|^{2} d x \\
& -\int_{\Omega} \frac{\partial \psi_{i}}{\partial x_{j}} \frac{\partial \psi_{i}}{\partial x_{k}} \frac{\partial h_{k}}{\partial x_{j}}
\end{aligned}
$$

and

$$
\begin{aligned}
\int_{\Omega} \frac{\partial \operatorname{div}(\psi)}{\partial x_{i}} h_{k} \frac{\partial \psi_{i}}{\partial x_{k}}= & \frac{1}{2} \int_{\Gamma} h \cdot \nu|\operatorname{div}(\psi)|^{2} d \Gamma+\frac{1}{2} \int_{\Omega} \operatorname{div}(h)|\operatorname{div}(\psi)|^{2} d x \\
& -\int_{\Omega} \operatorname{div}(\psi) \frac{\partial \psi_{i}}{\partial x_{k}} \frac{\partial h_{k}}{\partial x_{i}} .
\end{aligned}
$$

Here we have used the summation convention for repeated indices. It therefore follows that there exists a constant $c>0$ such that

$$
\begin{aligned}
\int_{\Gamma}\left[\mu\left|\frac{\partial \psi}{\partial \nu}\right|^{2}+(\lambda+\mu)|\operatorname{div}(\psi)|^{2}\right] d \Gamma & \\
& \leq c\left[\int_{\Omega}\left(\mu\left|\nabla \psi_{i}\right|^{2}+(\lambda+\mu)|\operatorname{div}(\psi)|^{2}\right) d x+\|f\|^{2}\right] .
\end{aligned}
$$

In addition, multiplying (2.31) by $\psi$ and integrating over $\Omega$ by parts, we deduce that there exists a constant $c>0$ such that

$$
\int_{\Omega}\left(\mu\left|\nabla \psi_{i}\right|^{2}+(\lambda+\mu)|\operatorname{div}(\psi)|^{2}\right) d x \leq c\|f\|^{2} .
$$

Hence, we have

$$
\int_{\Gamma}\left[\mu\left|\frac{\partial \psi}{\partial \nu}\right|^{2}+(\lambda+\mu)|\operatorname{div}(\psi)|^{2}\right] d \Gamma \leq c\|f\|^{2}
$$

which implies (2.33). Consequently, (2.28) follows from (2.32) and (2.33).

To prove inequality (2.29), we multiply $(2.27)$ by $u$ and $\varphi$, respectively, and integrate over $\Omega$ by parts. This gives

$$
\begin{aligned}
\int_{\Omega}[\mu \nabla u \cdot \nabla \varphi+(\lambda+\mu) \operatorname{div}(u) \operatorname{div}(\varphi)] d x & =\int_{\Gamma}\left[\mu \frac{\partial \varphi}{\partial \nu} \cdot u+(\lambda+\mu) \nu \cdot u \operatorname{div}(\varphi)\right] d \Gamma \\
& =\int_{\Omega}\left[\mu|\nabla \varphi|^{2}+(\lambda+\mu)|\operatorname{div}(\varphi)|^{2}\right] d x \\
& \geq 0 .
\end{aligned}
$$


The thermoelastic energy of (2.7) is defined by

$$
E(t)=E(u, \theta, t)=\frac{1}{2}\left\|\left(u(t), u^{\prime}(t), \theta(t)\right)\right\|_{\mathcal{H}}^{2} .
$$

By a straightforward calculation, we obtain

$$
E^{\prime}(t)=-\int_{\Gamma_{2}} m \cdot \nu g\left(u^{\prime}(t)\right) \cdot u^{\prime}(t) d \Gamma-\frac{\alpha}{\beta}\|\nabla \theta(t)\|^{2} .
$$

If $g$ satisfies that $g(u) \cdot u \geq 0$ for all $u \in \mathbf{R}^{n}$, then the energy $E(t)$ decreases in $(0, \infty)$.

What is more, we have the following decay rates. These are our main results of this paper.

Theorem 2.2. Let $\Gamma_{1}$ and $\Gamma_{2}$ be given by (2.1) and (2.2), respectively, satisfying

$$
\Gamma_{1} \cap \bar{\Gamma}_{2}=\emptyset \text {. }
$$

Suppose that (2.16) holds. Let the function $g \in\left(C\left(\mathbf{R}^{n}\right)\right)^{n}$ satisfy the following conditions:

$$
\begin{aligned}
g(0) & =0 \\
\left|g\left(u^{1}\right)-g\left(u^{2}\right)\right| & \leq k_{2}\left|u^{1}-u^{2}\right|^{q}, \quad \forall u^{1}, u^{2} \in \mathbf{R}^{n} \text { with }\left|u^{1}-u^{2}\right| \leq 1, \\
\left|g\left(u^{1}\right)-g\left(u^{2}\right)\right| & \leq k_{2}\left|u^{1}-u^{2}\right|, \quad \forall u^{1}, u^{2} \in \mathbf{R}^{n} \text { with }\left|u^{1}-u^{2}\right| \geq 1 \\
g(u) \cdot u & \geq k_{3}|u|^{p+1}, \quad \forall u \in \mathbf{R}^{n} \text { with }|u| \leq 1 \\
g(u) \cdot u & \geq k_{3}|u|^{2}, \quad \forall u \in \mathbf{R}^{n} \text { with }|u| \geq 1 \\
0 & \leq\left[g\left(u^{1}\right)-g\left(u^{2}\right)\right] \cdot\left(u^{1}-u^{2}\right), \quad \forall u^{1}, u^{2} \in \mathbf{R}^{n}
\end{aligned}
$$

for some constants $k_{2}, k_{3}>0$ and $p, q$ with $0<q \leq 1$. Suppose the function $a(x)$ satisfies

$$
2 K(a) R_{0} \gamma^{2}<1, \quad \text { for } n \leq 2,
$$

or

$$
a_{0} \leq \frac{(n-2) \mu}{2 R_{0}^{2}}, \quad \text { for } n \geq 3
$$

Then we have

(1) If $p=q=1$, there exist some constants $M \geq 1$ and $\omega>0$, independent of $\left(u^{0}, u^{1}, \theta^{0}\right)$, such that

$$
E(t) \leq M E(0) e^{-\omega t}, \quad \forall t \geq 0
$$

for all solutions of $(2.7)$ with $\left(u^{0}, u^{1}, \theta^{0}\right) \in \mathcal{H}$.

(2) If $p+1>2 q$, there exists $\tau>0$, depending on $\left(u^{0}, u^{1}, \theta^{0}\right)$, such that

$$
E(t) \leq 4 E(0)\left(1+\frac{p+1-2 q}{2 q} \tau t\right)^{-\frac{2 q}{p+1-2 q}}, \quad \forall t \geq 0
$$


for all solutions of $(2.7)$ with $\left(u^{0}, u^{1}, \theta^{0}\right) \in \mathcal{H}$. Further, the constants $M, \omega$ and $\tau$ can be explicitly given by

$$
\begin{aligned}
\sigma_{0} & =\frac{p+1-2 q}{2 q}, \\
\varepsilon & = \begin{cases}\left(1-2 K(a) R_{0} \gamma^{2}\right) / 8, & n \leq 2, \\
1 / 8, & n \geq 3,\end{cases} \\
C_{1} & =\frac{2 R_{0}}{\sqrt{\mu}}+(n-1) \lambda_{0}, \\
C_{2} & =\frac{\alpha^{2} R_{0}^{2}}{\mu \varepsilon}+\frac{\alpha^{2}(n-1)^{2} \lambda_{0}^{2}}{4 \varepsilon}+\frac{\alpha \lambda_{1}^{2}}{\beta} \\
C_{3} & =\frac{2 R_{0}^{2}}{\mu}+\frac{(n-1)^{2} R_{0} \gamma^{2}}{4 \varepsilon}, \\
C_{4} & =1+C_{3} k_{2}^{2}, \\
b_{1} & =\frac{1}{2} C_{1}^{-1} E^{-\sigma_{0}}(0), \\
b_{2} & =\alpha /\left[E^{\sigma_{0}}(0)\left(\sigma_{0} \alpha C_{1}+\beta C_{2}\right)\right], \\
b_{3} & =k_{3} /\left[E^{\sigma_{0}}(0)\left(C_{4}+\sigma_{0} C_{1} k_{3}\right)\right], \\
b_{4} & =k_{3}(p+1)^{(p+1) /(2 q)} /\left[C_{1} \sigma_{0} E^{\sigma_{0}}(0) k_{3}(p+1)^{(p+1) /(2 q)}\right. \\
\tau & \left.\quad+q\left(2 C_{4}\right)^{(p+1) /(2 q)}\left[R_{0} \operatorname{mes}\left(\Gamma_{2}\right)(p+1-2 q)\right]^{\sigma_{0}}\right], \\
\delta_{1} & =\min \left\{1 /\left(2 C_{1}\right), \alpha /\left(\beta C_{2}\right), k_{3} / C_{4}\right\}, \\
\delta_{2} & =\min \left\{b_{1}, b_{2}, b_{3}, b_{4}\right\}, \\
M & =\left(1+\delta_{1} C_{1}\right) /\left(1-\delta_{1} C_{1}\right) \leq 3, \\
\omega & =\delta_{1} /\left(1+\delta_{1} C_{1}\right), \\
& \left.\left.\left.=\delta_{2}(0)\right)^{-\sigma_{0}}\left[1+\delta_{2} C_{1} E^{\sigma_{0}}(0)\right]^{\sigma_{0}+1}\right]\right] .
\end{aligned}
$$

If the potential $a$ does not satisfy the smallness conditions (2.50) and (2.51), then we do not know whether or not Theorem 2.2 still holds. However, we can deal with large potentials $a$ under an additional condition on $\Gamma_{2}$. Namely, we have

Theorem 2.3. Assume that $\Gamma_{1}, \Gamma_{2}$ and $g$ satisfy the conditions of Theorem 2.2. We further assume that

$$
m \cdot \nu \geq \eta>0 \quad \text { on } \Gamma_{2}
$$

Suppose (2.16) holds. Then the decay properties (2.52) and (2.53) hold. 
Furthermore, the explicit values of the constants $M, \omega$ and $\tau$ are as follows:

$$
\begin{aligned}
\sigma_{0} & =\frac{p+1-2 q}{2 q} \\
\varepsilon & =1 / 8, \\
C_{0} & =\max \left\{0, \frac{2 a_{0} R_{0}^{2}}{\mu}+2-n\right\}, \\
C_{1}^{\prime} & =\frac{2 R_{0}}{\sqrt{\mu}}+(n-1) \lambda_{0}+C_{0} k_{1} \gamma, \\
C_{2}^{\prime} & =\frac{\alpha^{2} R_{0}^{2}}{\mu \varepsilon}+\frac{\alpha^{2}(n-1)^{2} \lambda_{0}^{2}}{4 \varepsilon}+\frac{C_{0}^{2} \alpha^{2} k_{1}^{2} \gamma^{2}}{4 \varepsilon}+\frac{\alpha \lambda_{1}^{2}}{\beta}, \\
C_{3}^{\prime} & =\frac{2 R_{0}^{2}}{\mu}+\frac{\left(n-1+C_{0}\right)^{2} R_{0} \gamma^{2}}{4 \varepsilon} \\
C_{4}^{\prime} & =R_{0}+\frac{C_{0}^{2} k_{1}^{2}}{4 \varepsilon}+C_{3}^{\prime} k_{2}^{2} R_{0}, \\
b_{1}^{\prime} & =\frac{1}{2} C_{1}^{\prime-1} E^{-\sigma_{0}}(0), \\
b_{2}^{\prime} & =\alpha /\left[E^{\sigma_{0}}(0)\left(\sigma_{0} \alpha C_{1}^{\prime}+\beta C_{2}^{\prime}\right)\right], \\
b_{3}^{\prime} & =k_{3} \eta /\left[E^{\sigma_{0}}(0)\left(C_{4}^{\prime}+\sigma_{0} C_{1}^{\prime} k_{3} R_{0}\right)\right], \\
b_{4}^{\prime} & =\eta k_{3}(p+1)^{(p+1) /(2 q)} /\left[C_{1}^{\prime} \sigma_{0} E^{\sigma_{0}}(0) R_{0} k_{3}(p+1)^{(p+1) /(2 q)}\right. \\
& \left.\quad+q\left[2 C_{4}^{\prime}\right]^{(p+1) /(2 q)}\left[\operatorname{mes}\left(\Gamma_{2}\right)(p+1-2 q)\right]^{\sigma_{0}}\right], \\
\delta_{1}^{\prime} & =\min \left\{1 /\left(2 C_{1}^{\prime}\right), \alpha /\left(\beta C_{2}^{\prime}\right), \eta k_{3} / C_{4}^{\prime}\right\}, \\
\delta_{2}^{\prime} & =\min \left\{b_{1}^{\prime}, b_{2}^{\prime}, b_{3}^{\prime}, b_{4}^{\prime}\right\}, \\
M & =\left(1+\delta_{1}^{\prime} C_{1}^{\prime}\right) /\left(1-\delta_{1}^{\prime} C_{1}^{\prime}\right) \leq 3, \\
\omega & =\delta_{1}^{\prime} /\left(1+\delta_{1}^{\prime} C_{1}^{\prime}\right), \\
\tau & \left.=\delta_{2}^{\prime} /\left[2(2 E(0))^{-\sigma_{0}}\left[1+\delta_{2}^{\prime} C_{1}^{\prime} E^{\sigma_{0}}(0)\right]^{\sigma_{0}+1}\right]\right] .
\end{aligned}
$$

EXAMPLE 2.4. It is easy to see that the following function

$$
g(u)=g\left(u_{1}, u_{2}, \ldots, u_{n}\right)= \begin{cases}\left(u_{1}^{2}+u_{2}^{2}+\cdots+u_{n}^{2}\right) u, & \text { if }|u| \leq 1 \\ u, & \text { if }|u| \geq 1\end{cases}
$$

satisfies the conditions of Theorem 2.2 with $p=3$ and any $0<q \leq 1$. In fact, (2.44), (2.46), (2.47) and (2.48) are obvious. In addition, it is easy to verify that $g$ is globally Lipschitz. Thus, for any $u^{1}, u^{2} \in \mathbf{R}^{n}$ with $\left|u^{1}-u^{2}\right| \leq 1$, we have

$$
\left|g\left(u^{1}\right)-g\left(u^{2}\right)\right| \leq c\left|u^{1}-u^{2}\right| \leq c\left|u^{1}-u^{2}\right|^{q} .
$$

To show (2.49), it suffices to show that the Jacobian matrix

$$
\left(\frac{\partial g_{i}}{\partial u_{j}}\right)=\left(\begin{array}{ccc}
\frac{\partial g_{1}}{\partial u_{1}} & \cdots & \frac{\partial g_{1}}{\partial u_{n}} \\
\vdots & & \vdots \\
\frac{\partial g_{n}}{\partial u_{1}} & \cdots & \frac{\partial g_{n}}{\partial u_{n}}
\end{array}\right)
$$


is positive semi-definite. If $|u| \geq 1$, this is obvious. If $|u| \leq 1$, then

$$
\left(\frac{\partial g_{i}}{\partial u_{j}}\right)=\left(\begin{array}{cccc}
3 u_{1}^{2} & 2 u_{1} u_{2} & \cdots & 2 u_{1} u_{n} \\
2 u_{1} u_{2} & 3 u_{2}^{2} & \cdots & 2 u_{2} u_{n} \\
\ldots & \cdots & \cdots & \cdots \\
2 u_{1} u_{n} & 2 u_{2} u_{n} & \cdots & 3 u_{n}^{2}
\end{array}\right)
$$

is also positive semi-definite since we have

$$
\begin{aligned}
\left|\begin{array}{cccc}
3 u_{1}^{2} & 2 u_{1} u_{2} & \cdots & 2 u_{1} u_{k} \\
2 u_{1} u_{2} & 3 u_{2}^{2} & \cdots & 2 u_{2} u_{k} \\
\cdots & \cdots & \cdots & \cdots \\
2 u_{1} u_{k} & 2 u_{2} u_{k} & \cdots & 3 u_{k}^{2}
\end{array}\right| & =u_{1}^{2} u_{2}^{2} \cdots u_{k}^{2}\left|\begin{array}{cccc}
3 & 2 & \cdots & 2 \\
2 & 3 & \cdots & 2 \\
\cdots & \cdots & \cdots & \cdots \\
2 & 2 & \cdots & 3
\end{array}\right| \\
& =u_{1}^{2} u_{2}^{2} \cdots u_{k}^{2}[3+2(k-1)] \\
& \geq 0 .
\end{aligned}
$$

On the other hand, if we take $g(u)=\left(h\left(u_{1}\right), \ldots, h\left(u_{n}\right)\right)$, where

$$
h(s)= \begin{cases}|s|^{p-1} s & \text { if }|s| \leq 1, \\ s, & \text { if }|s| \geq 1\end{cases}
$$

is a function on $\mathbf{R}$, then $g$ does not satisfy (2.47) and(2.48). But one can expect that this kind of functions should produce good decay rates. Therefore, we amend the conditions on $g$ in Theorems 2.2 and 2.3 and obtain the following theorem.

ThEOREM 2.5. Let $h(s)$ be a continuous function on $\mathbf{R}$ satisfying the following conditions:

$$
\begin{aligned}
h(0) & =0, \\
\left|h\left(s_{1}\right)-h\left(s_{2}\right)\right| & \leq k_{2}\left|s_{1}-s_{2}\right|^{q}, \quad \forall s_{1}, s_{2} \in \mathbf{R} \text { with }\left|s_{1}-s_{2}\right| \leq 1, \\
\left|h\left(s_{1}\right)-h\left(s_{2}\right)\right| & \leq k_{2}\left|s_{1}-s_{2}\right|, \quad \forall s_{1}, s_{2} \in \mathbf{R} \text { with }\left|s_{1}-s_{2}\right| \geq 1, \\
h(s) s & \geq k_{3}|s|^{p+1}, \quad \forall s \in \mathbf{R} \text { with }|s| \leq 1 \\
h(s) s & \geq k_{3}|s|^{2}, \quad \forall s \in \mathbf{R} \text { with }|s| \geq 1 \\
0 & \leq\left[h\left(s^{1}\right)-h\left(s^{2}\right)\right]\left(s^{1}-s^{2}\right), \quad \forall s^{1}, s^{2} \in \mathbf{R},
\end{aligned}
$$

for some constants $k_{2}, k_{3}>0$ and $p, q$ with $0<q \leq 1$. Suppose that

$$
\left.g(u)=h\left(u_{1}\right), \ldots, h\left(u_{n}\right)\right) .
$$

Assume that the conditions on $g$ in Theorem 2.2 are replaced by the above conditions and that the other conditions in Theorems 2.2 and 2.3 are kept unchanged. Then Theorems 2.2 and 2.3 still hold with the same constants except that $b_{4}$ is replaced by

$$
\begin{aligned}
k_{3}(p+1)^{(p+1) /(2 q)} /\left[C_{1} \sigma_{0} E^{\sigma_{0}}(0) k_{3}(p+1)^{(p+1) /(2 q)}\right. \\
\left.+q\left(2 C_{4}\right)^{(p+1) /(2 q)}\left[n R_{0} \operatorname{mes}\left(\Gamma_{2}\right)(p+1-2 q)\right]^{\sigma_{0}}\right],
\end{aligned}
$$

and $b_{4}^{\prime}$ is replaced by

$$
\begin{gathered}
\eta k_{3}(p+1)^{(p+1) /(2 q)} /\left[C_{1}^{\prime} \sigma_{0} E^{\sigma_{0}}(0) R_{0} k_{3}(p+1)^{(p+1) /(2 q)}\right. \\
\left.+q\left[2 C_{4}^{\prime}\right]^{(p+1) /(2 q)}\left[n \operatorname{mes}\left(\Gamma_{2}\right)(p+1-2 q)\right]^{\sigma_{0}}\right] .
\end{gathered}
$$


Furthermore, if $p>1$ and $q=1 / p$, then the decay rate (2.53) can be refined to

$$
E(t) \leq 4 E(0)\left(1+\frac{p-1}{2} \tau t\right)^{-\frac{2}{p-1}}, \quad \forall t \geq 0,
$$

with the same constants except the following changes:

$$
\begin{aligned}
\sigma_{0}= & \frac{p-1}{2}, \\
b_{4}= & (p+1)^{(p+1) / 2} /\left[C_{1} \sigma_{0} E^{\sigma_{0}}(0)(p+1)^{(p+1) / 2}\right. \\
& \left.\quad+\left(2 C_{5}\right)^{(p+1) / 2}\left[R_{0} \operatorname{mes}\left(\Gamma_{2}\right)(p-1)\right]^{\sigma_{0}}\right], \\
b_{4}^{\prime}= & \eta(p+1)^{(p+1) / 2} /\left[C_{1}^{\prime} \sigma_{0} E^{\sigma_{0}}(0) R_{0}(p+1)^{(p+1) / 2}\right. \\
& \left.\quad+\left[2 C_{5}^{\prime}\right]^{(p+1) / 2}\left[\operatorname{mes}\left(\Gamma_{2}\right)(p-1)\right]^{\sigma_{0}}\right], \\
C_{5}= & \frac{1+C_{3} k_{2} k_{3}}{k_{3}} \\
C_{5}^{\prime}= & \frac{4 \varepsilon R_{0}+C_{0} k_{1}^{2}+4 \varepsilon C_{3}^{\prime} k_{2} k_{3} R_{0}}{k_{3}} .
\end{aligned}
$$

REMARK 2.6. Note that condition (2.95) on $h$ implies that

$$
|h(s)| \geq k_{3}|s|^{p}, \quad \forall s \in \mathbf{R} \text { with }|s| \leq 1 .
$$

This means that $h(s)$ cannot degenerate at the origin faster than $|s|^{p}$. The following theorem provides a decay rate when the nonlinearity $h$ degenerates faster than any polynomial.

THEOREM 2.7. Let $h(s)$ be a continuous function on $\mathbf{R}$ satisfying the following conditions:

$$
\begin{aligned}
h(0) & =0, \\
\left|h\left(s_{1}\right)-h\left(s_{2}\right)\right| & \leq k_{2}\left|s_{1}-s_{2}\right|^{q}, \quad \forall s_{2}, s_{2} \in \mathbf{R} \text { with }\left|s_{1}-s_{2}\right| \leq 1, \\
\left|h\left(s_{1}\right)-h\left(s_{2}\right)\right| & \leq k_{2}\left|s_{1}-s_{2}\right|, \quad \forall s_{1}, s_{2} \in \mathbf{R} \text { with }\left|s_{1}-s_{2}\right| \geq 1, \\
h(s) s & \geq k_{3}|s|^{2}, \quad \forall s \in \mathbf{R} \text { with }|s| \geq 1, \\
0 & \leq\left[h\left(s^{1}\right)-h\left(s^{2}\right)\right]\left(s^{1}-s^{2}\right), \quad \forall s^{1}, s^{2} \in \mathbf{R},
\end{aligned}
$$

for some constants $k_{2}, k_{3}>0$ and $0<q \leq 1$. Suppose that

$$
g(u)=\left(h\left(u_{1}\right), \ldots, h\left(u_{n}\right)\right) .
$$

Assume that (2.16), (2.43) and (2.69) hold. Let $\varphi(s)$ denote an increasing and convex function defined on $[0, \infty]$ and twice differentiable outside $s=0$ such that $\varphi\left(s^{2 q}\right) \leq h(s) s$ on $[-1,1]$. Then the energy $E(t)$ of solutions of $(2.7)$ with $\left(u^{0}, u^{1}, \theta^{0}\right) \in \mathcal{H}$ satisfies the following decay rate:

$$
E(t) \leq 2 V(t), \quad \text { for } t \geq 0,
$$

where $V(t)$ is the solution of the following differential equation:

$$
V^{\prime}(t)=-\frac{\delta V(t)}{2 b} \varphi^{\prime}\left(\frac{a V(t)}{b}\right)-n \delta\left(R_{0}+2 C_{0}^{2} k_{1}^{2}+C_{3}^{\prime} k_{2}^{2} R_{0}\right) \operatorname{mes}\left(\Gamma_{2}\right) \varphi\left(\frac{a V(t)}{b}\right),
$$


where $\delta$ is a sufficiently small positive constant and

$$
\begin{aligned}
a & =\frac{1}{2 n\left(R_{0}+2 C_{0}^{2} k_{1}^{2}+C_{3}^{\prime} k_{2}^{2} R_{0}\right) \operatorname{mes}\left(\Gamma_{2}\right)}, \\
b & =1+\delta C_{1}^{\prime} \varphi^{\prime}(a E(0)) .
\end{aligned}
$$

Furthermore, we have

$$
\lim _{t \rightarrow \infty} E(t)=\lim _{t \rightarrow \infty} V(t)=0 .
$$

REMARK 2.8. The function $\varphi$ that satisfies the conditions of Theorem 2.7 always exists. For example, we set

$$
\bar{\varphi}(s)=\operatorname{conv}\left[\min \left\{s^{1 /(2 q)} h\left(s^{1 /(2 q)}\right),-s^{1 /(2 q)} h\left(-s^{1 /(2 q)}\right)\right\}\right], \quad \text { for } 0 \leq s \leq 1,
$$

and extend it to $[0, \infty)$. Here conv denotes the convex envelope of a function. Then we can take an increasing, convex and twice differentiable function $\varphi(s)$ such that $\varphi(s) \leq \bar{\varphi}(s)$.

In the special case where $s^{1 /(2 q)} h\left(s^{1 /(2 q)}\right)$ is convex and $h(s)$ is odd, we have:

Corollary 2.9. Assume that all the conditions of Theorem 2.7 hold. If, further, $h$ is odd in $[-1,1]$ and $s^{1 /(2 q)} h\left(s^{1 /(2 q)}\right)$ is convex on $[0,1]$, then the energy $E(t)$ of $(2.7)$ satisfies the following decay rate:

$$
E(t) \leq 2 V(t), \quad \text { for } t \geq 0
$$

where, for $t$ large enough, $V(t)$ satisfies the following differential equation:

$$
V^{\prime}(t)=-K_{1} V^{1 /(2 q)} h\left(\left(\frac{a V}{b}\right)^{1 /(2 q)}\right)-K_{2} V^{1 / q} h^{\prime}\left(\left(\frac{a V}{b}\right)^{1 /(2 q)}\right)
$$

where $K_{1}, K_{2}$ are positive constants independent of $V$.

Corollary 2.10. Assume that all the conditions of Theorem 2.7 hold. If, further, $h$ satisfies (2.95), then the decay properties (2.52) and (2.53) hold.

Corollary 2.10 shows that Theorem 2.5 is covered by Theorem 2.7 when (2.69) holds. Since Theorem 2.7 does not include the case where (2.69) does not hold, we separate it from Theorem 2.7 .

We now give an example of logarithmic decay rate which complements the example of polynomial decay rate existing in the literature.

ExAmPle 2.11. Logarithmic Decay Rate. Let $h(s)$ satisfy

$$
\begin{aligned}
h(s) & =s^{3} e^{-1 / s^{2}}, \quad|s| \leq 1, \\
c_{1}|s| \leq h(s) & \leq|s|, \quad|s| \geq 1 .
\end{aligned}
$$

It is easy to see that $h$ satisfies all the conditions of Corollary 2.9 with $q=1$. Consequently, for $t$ large enough, by (2.121), $V$ satisfies

$$
V^{\prime}(t) \leq-\omega V^{2} e^{-\frac{b}{a V}}
$$

which is the same as

$$
\left(e^{\frac{b}{a V}}\right)^{\prime} \geq \frac{b \omega}{a}
$$


where $\omega$ is a positive constant independent of $V$. Solving the inequality, we obtain the logarithmic decay rate

$$
V(t) \leq \frac{b}{a}\left[\log \left(\frac{b \omega}{a} t+e^{\frac{b}{a V(0)}}\right)\right]^{-1} .
$$

REMARK 2.12. The decay rate of the form (2.101) has been established for the wave equation [21, p. 127] and the compactly coupled wave equations [22]. It can be seen from the proof of Theorem 2.5 at the end of section 4 that the key point to obtain this decay rate $t^{-2 /(p-1)}$ is to enlarge the following inequality:

$$
s^{2}+|h(s)|^{2} \leq c(h(s) s)^{2 /(p+1)} .
$$

This can be done as follows. We first deduce from (2.95) that

$$
s^{2} \leq c(h(s) s)^{2 /(p+1)} .
$$

Next, we have

$$
\begin{aligned}
|h(s)| & \leq c|s|^{1 / p} \\
|h(s)|^{2 p /(p+1)} & \leq c|s|^{2 /(p+1)}, \\
|h(s)|^{(2 p+2-2) /(p+1)} & \leq c|s|^{2 /(p+1)}, \\
|h(s)|^{2} & \leq\left. c|s|^{2 /(p+1)}\right|^{2 /(p+1)}|h(s)|^{2 /(p+1)}=c|h(s) s|^{2 /(p+1)} .
\end{aligned}
$$

In the situation of Theorems 2.2 and 2.3 , since, for the vector function $g(u)$ and vector $u$, we have

$$
|u|^{2 /(p+1)}|g(u)|^{2 /(p+1)} \geq|u \cdot g(u)|^{2 /(p+1)},
$$

the analogous inequality of (2.127) for $g$ no longer holds. Thus, the decay rate (2.53) of Theorems 2.2 and 2.3 cannot be refined to (2.101) in the case where $p>1$ and $q=1 / p$.

REMARK 2.13. Observe that (2.44), (2.45) and (2.47) imply that $q \geq p$. Therefore, under the assumption $0<q \leq 1$, we have $2 q \leq p+1$. If $2 q=p+1$, then $q-1=p-q \geq 0$. Hence we have $q=p=1$. Therefore, we have only two cases: $q=p=1$ and $2 q<p+1$, as stated in Theorem 2.2.

REMARK 2.14. Under condition (2.43), assumptions (2.1) and (2.2) imply that the domain $\Omega$ is simply connected and star-shaped with respect to $x^{0} \in \Omega$ (in which case $\left.\Gamma_{1}=\emptyset\right)$ or $\Omega=\Omega_{1}-\bar{\Omega}_{2}$, both $\Omega_{1}$ and $\Omega_{2}$ being star-shaped with respect to $x^{0}$. If $\Gamma_{1} \cap \bar{\Gamma}_{2} \neq \emptyset$, it is well known that the solution of (2.7) is not regular enough (see [14]) to perform the integrations by parts that we will do later. Thus, the obtention of decay rates in this case is an open problem. The extension of the results of this paper to the case $\Gamma_{1} \cap \bar{\Gamma}_{2} \neq \emptyset$ requires a careful analysis of the singularities that the solution may develop on $\Gamma_{1} \cap \bar{\Gamma}_{2}$ as in [16].

REMARK 2.15. The expressions of $\omega$ and $\tau$ in the decay rates may look complex. But they are useful since they provide explicit estimates of the dependence of the decay rates $\omega$ and $\tau$ on the various parameters $\alpha, \beta, \Omega, k_{2}, k_{3}, \ldots$, etc. For example, if we take a linear boundary feedback $g(s)=k_{3} s$ and let $k_{3} \rightarrow 0$, then it follows from (2.64) and (2.67) that $\omega \rightarrow 0$. In addition, by these expressions, we can analyze the limit of the polynomial 
decay rate $\left(1+\frac{p+1-2 q}{2 q} \tau t\right)^{-\frac{2 q}{p+1-2 q}}$ as $p, q$ tend to 1 and recover the exponential decay of the case $p=q=1$. Indeed, it is easy to see that

$$
\begin{gathered}
\lim _{p, q \rightarrow 1} \delta_{2}(p, q)=\min \left\{\frac{1}{2} C_{1}, \alpha /\left(\beta C_{2}\right), k_{3} / C_{4}\right\}=\delta_{1}, \\
\lim _{p, q \rightarrow 1} \tau(p, q)=\delta_{1}\left[2\left(1+\delta_{1} C_{1}\right)\right]=\omega / 2, \\
\lim _{p, q \rightarrow 1}\left(1+\frac{p+1-2 q}{2 q} \tau t\right)^{-\frac{2 q}{p+1-2 q}}=e^{-\omega t / 2} .
\end{gathered}
$$

REMARK 2.16. For the linear elastodynamic system, the uniform stabilization with the boundary feedback of the form

$$
\sigma_{i j}(u) \nu_{j}=u_{i}^{\prime}
$$

was established by Horn [18] by developing microlocal estimates (see [19]) for tangential derivatives of the solutions of the elastodynamic system. However, this remains to be done for the system of thermoelasticity.

3. Well-posedness. In this section, we use the theory of nonlinear semigroups to treat the problem of well-posedness of (2.7). Therefore, we formulate (2.7) as an abstract Cauchy problem.

Let $\langle\cdot, \cdot\rangle$ denote the duality pairing between $\left(H_{\Gamma_{1}}^{1}(\Omega)\right)^{n}$ and $\left[\left(H_{\Gamma_{1}}^{1}(\Omega)\right)^{n}\right]^{\prime}$ or $H_{0}^{1}(\Omega)$ and $H^{-1}(\Omega)$. We define the duality operator $A:\left(H_{\Gamma_{1}}^{1}(\Omega)\right)^{n} \rightarrow\left[\left(H_{\Gamma_{1}}^{1}(\Omega)\right)^{n}\right]^{\prime}$ by

$$
\langle A u, v\rangle=(u, v)_{\left(H_{\Gamma_{1}}^{1}(\Omega)\right)^{n}}, \quad \forall u, v \in\left(H_{\Gamma_{1}}^{1}(\Omega)\right)^{n},
$$

and the duality operator $A_{0}: H_{0}^{1}(\Omega) \rightarrow H^{-1}(\Omega)$ by

$$
\left\langle A_{0} u, v\right\rangle=(u, v)_{H_{0}^{1}(\Omega)}, \quad \forall u, v \in H_{0}^{1}(\Omega)
$$

The Riesz representation theorem ensures that $A$ and $A_{0}$ are isometric isomorphisms of $\left(H_{\Gamma_{1}}^{1}(\Omega)\right)^{n}$ onto $\left[\left(H_{\Gamma_{1}}^{1}(\Omega)\right)^{n}\right]^{\prime}$ and $H_{0}^{1}(\Omega)$ onto $H^{-1}(\Omega)$, respectively.

Also, we define a nonlinear operator $B$ by

$$
\langle B u, v\rangle=\int_{\Gamma_{2}} m \cdot \nu g(u) \cdot v d \Gamma, \quad \forall u, v \in\left(H_{\Gamma_{1}}^{1}(\Omega)\right)^{n} .
$$

LEMma 3.1. Suppose that the function $g \in\left(C\left(\mathbf{R}^{n}\right)\right)^{n}$. If there exist constants $c>0$ and $\sigma \geq 0$ such that for $u \in \mathbf{R}^{n}$

$$
|g(u)| \leq \begin{cases}c\left[1+|u|^{n /(n-2)},\right. & n \geq 3 \\ c\left(1+|u|^{\sigma}\right), & n=2\end{cases}
$$

then $B$ maps $\left(H_{\Gamma_{1}}^{1}(\Omega)\right)^{n}$ into $\left[\left(H_{\Gamma_{1}}^{1}(\Omega)\right)^{n}\right]^{\prime}$. Furthermore, $B$ is hemicontinuous, that is, we have

$$
\lim _{t \rightarrow 0}\left\langle B\left(u^{1}+t u^{2}\right), v\right\rangle=\left\langle B u^{1}, v\right\rangle
$$

for any $u^{1}, u^{2}, v \in\left(H_{\Gamma_{1}}^{1}(\Omega)\right)^{n}$. 
Proof. If $n \geq 3$, then, by the trace theorem (see [36, Chap. 1]) and the imbedding theorem (see [1, p. 217], we have

$$
H_{\Gamma_{1}}^{1}(\Omega) \hookrightarrow H^{1 / 2}(\Gamma) \subset L^{(2 n-2) /(n-2)}(\Gamma) .
$$

Thus, we have

$$
\begin{aligned}
|\langle B u, v\rangle| & =\left|\int_{\Gamma_{2}} m \cdot \nu g(u) \cdot v d \Gamma\right| \\
& \leq c\left(\int_{\Gamma_{2}}|g(u)|^{(2 n-2) / n} d \Gamma\right)^{n /(2 n-2)}\left(\int_{\Gamma_{2}}|v|^{(2 n-2) /(n-2)} d \Gamma\right)^{(n-2) /(2 n-2)} \\
& \leq c\left[1+\left(\int_{\Gamma_{2}}|u|^{(2 n-2) /(n-2)} d \Gamma\right)^{n /(2 n-2)}\right]\|v\|_{\left(H_{\Gamma_{1}}^{1}(\Omega)\right)^{n}} \\
& \leq c\left[1+\|u\|_{\left(H_{\Gamma_{1}}^{1}(\Omega)\right)^{n}}^{n /(n-2)}\right]\|v\|_{\left(H_{\Gamma_{1}}^{1}(\Omega)\right)^{n}} .
\end{aligned}
$$

This shows that $B u \in\left[\left(H_{\Gamma_{1}}^{1}(\Omega)\right)^{n}\right]^{\prime}$.

If $n=2$, then, by the trace theorem (see [36, Chap. 1]) and the imbedding theorem (see $[1$, p. 217]), we have

$$
H_{\Gamma_{1}}^{1}(\Omega) \hookrightarrow H^{1 / 2}(\Gamma) \subset L^{r}(\Gamma)
$$

for any $2 \leq r<\infty$. In addition, we may as well assume that $\sigma \geq 1$ since, by condition (3.4), we have

$$
|g(u)| \leq 2 c \leq 2 c\left(1+|u|^{\sigma+1}\right), \quad \text { for }|u| \leq 1,
$$

and

$$
|g(u)| \leq c\left(1+|u|^{\sigma+1}\right) \leq 2 c\left(1+|u|^{\sigma+1}\right), \quad \text { for }|u| \geq 1 .
$$

It therefore follows that

$$
\begin{aligned}
|\langle B u, v\rangle| & =\left|\int_{\Gamma_{2}} m \cdot \nu g(u) \cdot v d \Gamma\right| \\
& \leq c\left(\int_{\Gamma_{2}}|g(u)|^{2} d \Gamma\right)^{1 / 2}\left(\int_{\Gamma_{2}}|v|^{2} d \Gamma\right)^{1 / 2} \\
& \leq c\left[1+\left(\int_{\Gamma_{2}}|u|^{2 \sigma} d \Gamma\right)^{1 / 2}\right]\|v\|_{\left(H_{\Gamma_{1}}^{1}(\Omega)\right)^{n}} \\
& \leq c\left[1+\|u\|_{\left(H_{\Gamma_{1}}^{1}(\Omega)\right)^{n}}^{\sigma}\right]\|v\|_{\left(H_{\Gamma_{1}}^{1}(\Omega)\right)^{n}} .
\end{aligned}
$$

This shows that $B u \in\left[\left(H_{\Gamma_{1}}^{1}(\Omega)\right)^{n}\right]^{\prime}$.

If $n=1$, this is just the consequence of the following embedding:

$$
H_{\Gamma_{1}}^{1}(\Omega) \hookrightarrow C(\bar{\Omega}) .
$$

It remains to prove that $B$ is hemicontinuous. By the continuity of $g$, we deduce that for any $u^{1}, u^{2}, v \in\left(H_{\Gamma_{1}}^{1}(\Omega)\right)^{n}$

$$
g\left(u^{1}+t u^{2}\right) \rightarrow g\left(u^{1}\right) \quad \text { as } t \rightarrow 0 \text { a.e. on } \Gamma .
$$


It therefore follows from Lebesgue's dominated convergence theorem and (3.4) that

$$
\begin{aligned}
\lim _{t \rightarrow 0}\left\langle B\left(u^{1}+t u^{2}\right), v\right\rangle & =\int_{\Gamma_{2}} m \cdot \nu g\left(u^{1}+t u^{2}\right) \cdot v d \Gamma \\
& =\int_{\Gamma_{2}} m \cdot \nu g\left(u^{1}\right) \cdot v d \Gamma \\
& =\left\langle B u^{1}, v\right\rangle .
\end{aligned}
$$

This shows that $B$ is hemicontinuous.

Note that if $g$ satisfies (2.45) and (2.46) of Theorem 2.2 (or (2.93) and (2.94) of Theorem 2.5), then $g$ satisfies (3.4). Thus $B$ maps $\left(H_{\Gamma_{1}}^{1}(\Omega)\right)^{n}$ into $\left[\left(H_{\Gamma_{1}}^{1}(\Omega)\right)^{n}\right]^{\prime}$.

Using the operators $A, A_{0}$ and $B$, we can formally transform problem (2.7) into an abstract Cauchy problem. In doing so, we multiply the first equation of (2.7) by $v \in\left(H_{\Gamma_{1}}^{1}(\Omega)\right)^{n}$ and integrate over $\Omega$ by parts. This gives

$$
\begin{aligned}
0= & \int_{\Omega}\left(u^{\prime \prime}-\mu \Delta u-(\lambda+\mu) \nabla \operatorname{div} u+\alpha \nabla \theta\right) \cdot v d x \\
= & \int_{\Omega} u^{\prime \prime} \cdot v d x-\mu \int_{\Gamma} \frac{\partial u}{\partial \nu} \cdot v d \Gamma-(\lambda+\mu) \int_{\Gamma} v \cdot \nu \operatorname{div}(u) d \Gamma \\
& +\int_{\Omega}(\mu \nabla u \cdot \nabla v+(\lambda+\mu) \operatorname{div} u \operatorname{div} v) d x+\int_{\Omega} \alpha \nabla \theta \cdot v d x \\
= & \int_{\Omega} u^{\prime \prime} \cdot v d x+\int_{\Gamma} a m \cdot \nu u \cdot v d \Gamma+\int_{\Gamma} m \cdot \nu g\left(u^{\prime}\right) \cdot v d \Gamma \\
& +\int_{\Omega}(\mu \nabla u \cdot \nabla v+(\lambda+\mu) \operatorname{div} u \operatorname{div} v) d x+\int_{\Omega} \alpha \nabla \theta \cdot v d x \\
= & \left\langle u^{\prime \prime}, v\right\rangle+\langle A u, v\rangle+\left\langle B u^{\prime}, v\right\rangle+\langle\alpha \nabla \theta, v\rangle,
\end{aligned}
$$

and therefore,

$$
u^{\prime \prime}+A u+B u^{\prime}+\alpha \nabla \theta=0 .
$$

Similarly, multiplying the second equation of $(2.7)$ by $v \in H_{0}^{1}(\Omega)$ and integrating over $\Omega$ by parts, we obtain

$$
\theta^{\prime}+A_{0} \theta+\beta \operatorname{div} u^{\prime}=0
$$

Setting

$$
\begin{aligned}
\Phi & =\left(u, u^{\prime}, \theta\right), \\
\Lambda \Phi & =\left(-u^{\prime}, A u+B u^{\prime}+\alpha \nabla \theta, A_{0} \theta+\beta \operatorname{div} u^{\prime}\right),
\end{aligned}
$$

we transform (2.7) into

$$
\left\{\begin{array}{l}
\Phi^{\prime}+\Lambda \Phi=0, \\
\Phi(0)=\left(u^{0}, u^{1}, \theta^{0}\right) .
\end{array} \quad t>0\right.
$$

This leads us to define the solution of (2.7) as that of (3.20). 
Consider the nonlinear operator $\Lambda$ on $\mathcal{H}=\left(H_{\Gamma_{1}}^{1}(\Omega)\right)^{n} \times\left(L^{2}(\Omega)\right)^{n} \times L^{2}(\Omega)$ with the domain

$$
\begin{array}{r}
D(\Lambda)=\left\{(u, v, \theta) \in \mathcal{H}: v \in\left(H_{\Gamma_{1}}^{1}(\Omega)\right)^{n}, A u+B v \in\left(L^{2}(\Omega)\right)^{n}\right. \\
\left.\theta \in H^{2}(\Omega) \cap H_{0}^{1}(\Omega)\right\} .
\end{array}
$$

We are going to prove that $-\Lambda$ is $m$-dissipative. For the definition of $m$-dissipativeness, we refer to $[6$, p. 71$]$.

In what follows, we denote by $(\cdot, \cdot)$ the inner product in $L^{2}(\Omega)$ or $\left(L^{2}(\Omega)\right)^{n}$.

Lemma 3.2. Suppose that $g$ satisfies (2.44), (2.49) (or (2.92), (2.97)) and (3.4). Then the operator $-\Lambda$ is $m$-dissipative on $\mathcal{H}$.

Proof. By (2.49) (or (2.97)), we obtain that for any $\left(u^{1}, v^{1}, \theta^{1}\right),\left(u^{2}, v^{2}, \theta^{2}\right) \in D(\Lambda)$

$$
\begin{aligned}
&\left(\Lambda\left(u^{1}, v^{1}, \theta^{1}\right)-\Lambda\left(u^{2}, v^{2}, \theta^{2}\right),\left(u^{1}, v^{1}, \theta^{1}\right)-\left(u^{2}, v^{2}, \theta^{2}\right)\right)_{\mathcal{H}} \\
&=\left(v^{2}-v^{1}, u^{1}-u^{2}\right)_{\left(H_{\Gamma_{1}}^{1}(\Omega)\right)^{n}}+\frac{\alpha}{\beta}\left(A_{0}\left(\theta^{1}-\theta^{2}\right)+\beta \operatorname{div}\left(v^{1}-v^{2}\right), \theta^{1}-\theta^{2}\right) \\
& \quad+\left(A\left(u^{1}-u^{2}\right)+B v^{1}-B v^{2}+\alpha \nabla\left(\theta^{1}-\theta^{2}\right), v^{1}-v^{2}\right) \\
&= \int_{\Gamma_{2}} m \cdot \nu\left[g\left(v^{1}\right)-g\left(v^{2}\right)\right] \cdot\left(v^{1}-v^{2}\right) d \Gamma+\frac{\alpha}{\beta}\left\|\nabla\left(\theta^{1}-\theta^{2}\right)\right\|^{2} \\
& \geq 0 .
\end{aligned}
$$

Thus, $-\Lambda$ is dissipative.

It remains to show that

$$
(I+\Lambda)(D(\Lambda))=\mathcal{H}
$$

Namely, we want to prove that

$$
\left\{\begin{array}{l}
u-v=\varphi, \\
v+A u+B v+\alpha \nabla \theta=\psi, \\
\theta+A_{0} \theta+\beta \operatorname{div} v=\xi
\end{array}\right.
$$

has a solution $(u, v, \theta) \in D(\Lambda)$ for every $(\varphi, \psi, \xi) \in \mathcal{H}$. For this, it suffices to prove that the following problem

$$
\left\{\begin{array}{l}
v+A v+B v+\alpha \nabla \theta=\psi-A \varphi, \\
\theta+A_{0} \theta+\beta \operatorname{div} v=\xi
\end{array}\right.
$$

has a solution $v \in\left(H_{\Gamma_{1}}^{1}(\Omega)\right)^{n}$ and $\theta \in H^{2}(\Omega) \cap H_{0}^{1}(\Omega)$ for every $(\varphi, \psi, \xi) \in \mathcal{H}$. In fact, if this has been done, then, by setting $u=v+\varphi$, it is easy to see that $(u, v, \theta)$ satisfies (3.24). Further, we have

$$
A u+B v=\psi-v-\alpha \nabla \theta \in\left(L^{2}(\Omega)\right)^{n} .
$$

Thus, we have $(u, v, \theta) \in D(\Lambda)$.

To prove that (3.25) has a required solution, it suffices to show that the nonlinear operator $\mathcal{A}$ defined by

$$
\mathcal{A}(v, \theta)=\left(v+A v+B v+\alpha \nabla \theta, \frac{\alpha}{\beta} \theta+\frac{\alpha}{\beta} A_{0} \theta+\alpha \operatorname{div} v\right)
$$


$\operatorname{maps}\left(H_{\Gamma_{1}}^{1}(\Omega)\right)^{n} \times H_{0}^{1}(\Omega)$ onto $\left[\left(H_{\Gamma_{1}}^{1}(\Omega)\right)^{n}\right]^{\prime} \times H^{-1}(\Omega)$, that is,

$$
\mathcal{A}\left(\left(H_{\Gamma_{1}}^{1}(\Omega)\right)^{n} \times H_{0}^{1}(\Omega)\right)=\left[\left(H_{\Gamma_{1}}^{1}(\Omega)\right)^{n}\right]^{\prime} \times H^{-1}(\Omega) .
$$

In fact, if this has been done, then, since $\psi-A \varphi \in\left[\left(H_{\Gamma_{1}}^{1}(\Omega)\right)^{n}\right]^{\prime}$ and $\frac{\alpha}{\beta} \xi \in L^{2}(\Omega)$, there exist $(v, \theta) \in\left(H_{\Gamma_{1}}^{1}(\Omega)\right)^{n} \times H_{0}^{1}(\Omega)$ such that (3.25) holds. Further, since $A_{0} \theta=$ $\xi-\theta-\beta \operatorname{div} v \in L^{2}(\Omega)$, we have $\theta \in H^{2}(\Omega) \cap H_{0}^{1}(\Omega)$.

To prove (3.28), by Theorem 1.3 of $[6$, p. 40], it suffices to show $\mathcal{A}$ is monotone, coercive and hemicontinuous. For any $\left(v^{1}, \theta^{1}\right),\left(v^{2}, \theta^{2}\right) \in\left(H_{\Gamma_{1}}^{1}(\Omega)\right)^{n} \times H_{0}^{1}(\Omega)$, we have

$$
\begin{aligned}
&\left\langle\mathcal{A}\left(v^{1}, \theta^{1}\right)-\mathcal{A}\left(v^{2}, \theta^{2}\right),\left(v^{1}, \theta^{1}\right)-\left(v^{2}, \theta^{2}\right)\right\rangle \\
&=\left\langle v^{1}-v^{2}, v^{1}-v^{2}\right\rangle+\left\langle A\left(v^{1}-v^{2}\right), v^{1}-v^{2}\right\rangle+\left\langle B v^{1}-B v^{2}, v^{1}-v^{2}\right\rangle \\
&+\left\langle\alpha \nabla\left(\theta^{1}-\theta^{2}\right), v^{1}-v^{2}\right\rangle+\frac{\alpha}{\beta}\left\langle\theta^{1}-\theta^{2}, \theta^{1}-\theta^{2}\right\rangle \\
& \quad+\frac{\alpha}{\beta}\left\langle A_{0}\left(\theta^{1}-\theta^{2}\right), \theta^{1}-\theta^{2}\right\rangle+\alpha\left\langle\operatorname{div}\left(v^{1}-v^{2}\right), \theta^{1}-\theta^{2}\right\rangle \\
& \geq 0 .
\end{aligned}
$$

So $\mathcal{A}$ is monotone. Similarly, we have

$$
\begin{aligned}
\langle\mathcal{A}(v, \theta),(v, \theta)\rangle= & \langle v, v\rangle+\langle A v, v\rangle+\langle B v, v\rangle \\
& +\frac{\alpha}{\beta}\langle\theta, \theta\rangle+\frac{\alpha}{\beta}\left\langle A_{0} \theta, \theta\right\rangle \\
& \geq\|v\|_{\left.H_{\Gamma_{1}}^{1}(\Omega)\right)^{n}}^{2}+\frac{\alpha}{\beta}\|\theta\|_{\left.H_{0}^{1} \Omega\right)}^{2}
\end{aligned}
$$

Thus $\mathcal{A}$ is coercive. On the other hand, by Lemma 3.1 , we can readily deduce that $\mathcal{A}$ is hemicontinuous. That is, we have

$$
\lim _{t \rightarrow 0}\left\langle\mathcal{A}\left[\left(v^{1}, \theta^{1}\right)+t\left(v^{2}, \theta^{2}\right)\right],(v, \theta)\right\rangle=\left\langle\mathcal{A}\left(v^{1}, \theta^{1}\right),(v, \theta)\right\rangle
$$

for any $\left(v^{1}, \theta^{1}\right),\left(v^{2}, \theta^{2}\right),(v, \theta) \in\left(H_{\Gamma_{1}}^{1}(\Omega)\right)^{n} \times H_{0}^{1}(\Omega)$. This completes the proof.

Lemma 3.3. Suppose that (2.43) holds. If $g$ satisfies (2.44) and (3.4), then $D(\Lambda)$ is dense in $\mathcal{H}$. Further, if $g$ satisfies (2.45) and (2.46) (or (2.93) and (2.94)), we have

$$
\begin{gathered}
D(\Lambda) \subset D_{0}=\left\{(u, v, \theta) \in \mathcal{H}: u \in\left(H^{s}(\Omega) \cap H_{\Gamma_{1}}^{1}(\Omega)\right)^{n}, v \in\left(H_{\Gamma_{1}}^{1}(\Omega)\right)^{n},\right. \\
\theta \in H^{2}(\Omega) \cap H_{0}^{1}(\Omega), \\
\left.\mu \frac{\partial u}{\partial \nu}+(\lambda+\mu) \nu \operatorname{div} u+a m \cdot \nu u+m \cdot \nu g(v)=0 \text { on } \Gamma_{2}\right\},
\end{gathered}
$$

for some $s>3 / 2$.

Proof. Set

$$
\begin{aligned}
& D=\left\{(u, v, \theta) \in \mathcal{H}: u \in\left(H^{2}(\Omega)\right.\right.\left.\cap H_{\Gamma_{1}}^{1}(\Omega)\right)^{n}, v \in\left(H_{0}^{1}(\Omega)\right)^{n}, \\
& \theta \in H^{2}(\Omega) \cap H_{0}^{1}(\Omega), \\
&\left.\mu \frac{\partial u}{\partial \nu}+(\lambda+\mu) \nu \operatorname{div} u+a m \cdot \nu u=0 \text { on } \Gamma_{2}\right\} .
\end{aligned}
$$


To prove that $D(\Lambda)$ is dense in $\mathcal{H}$, it suffices to prove that $D$ is dense in $\mathcal{H}$ and $D \subset D(\Lambda)$. Firstly, we prove that $D$ is dense in $\mathcal{H}$. For this, it suffices to prove that

$$
W=\left\{w \in\left(H^{2}(\Omega) \cap H_{\Gamma_{1}}^{1}(\Omega)\right)^{n}: \mu \frac{\partial w}{\partial \nu}+(\lambda+\mu) \nu \operatorname{div} w+a m \cdot \nu w=0 \quad \text { on } \Gamma_{2}\right\}
$$

is dense in $\left(H_{\Gamma_{1}}^{1}(\Omega)\right)^{n}$. Let $v \in\left(H_{\Gamma_{1}}^{1}(\Omega)\right)^{n}$ be such that

$$
(v, w)_{\left(H_{\Gamma_{1}}^{1}(\Omega)\right)^{n}}=0, \quad w \in W .
$$

For any fixed $f \in\left(L^{2}(\Omega)\right)^{n}$, we consider the following elliptic problem:

$$
\begin{cases}-\mu \Delta u-(\lambda+\mu) \nabla \operatorname{div} u=f & \text { in } \Omega, \\ u=0, & \text { on } \Gamma_{1}, \\ \mu \frac{\partial u}{\partial \nu}+(\lambda+\mu) \nu \operatorname{div} u+a m \cdot \nu u=0 & \text { on } \Gamma_{2} .\end{cases}
$$

By the elliptic regularity theory, problem (3.36) has a solution $u \in W$. Thus, by (3.35), we have

$$
(v, f)=(v, u)_{\left(H_{\Gamma_{1}}^{1}(\Omega)\right)^{n}}=0 .
$$

Hence, we deduce that $v=0$. It therefore follows from the Hahn-Banach theorem that $W$ is dense in $\left(H_{\Gamma_{1}}^{1}(\Omega)\right)^{n}$.

Next, we prove that $D \subset D(\Lambda)$. Let $(u, v, \theta) \in D$. To prove that $(u, v, \theta) \in D(\Lambda)$, it suffices to prove that $A u+B v \in\left(L^{2}(\Omega)\right)^{n}$. For this, let $w \in\left(H_{\Gamma_{1}}^{1}(\Omega)\right)^{n}$. By the definition of $A$ and $B$, we have

$$
\begin{aligned}
\langle A u+B v, w\rangle= & (u, w)_{\left(H_{\Gamma_{1}}^{1}(\Omega)\right)^{n}}+\int_{\Gamma_{2}} m \cdot \nu g(v) \cdot w d \Gamma \\
= & \int_{\Omega}(\mu \nabla u \cdot \nabla w+(\lambda+\mu) \operatorname{div} u \operatorname{div} w) d x \\
& +\int_{\Gamma_{2}} a m \cdot \nu u \cdot w d \Gamma \quad(\text { use } g(0)=0) \\
= & -\int_{\Omega}(\mu \Delta u+(\lambda+\mu) \nabla \operatorname{div} u) \cdot w d x,
\end{aligned}
$$

since $u \in\left(H^{2}(\Omega)\right)^{n}$. It therefore follows that there exists a constant $c$, depending on $u$, such that

$$
|\langle A u+B v, w\rangle| \leq c\|w\|,
$$

which implies that $A u+B v \in\left(L^{2}(\Omega)\right)^{n}$. Thus $(u, v, \theta) \in D(\Lambda)$.

It remains to prove that $D(\Lambda) \subset D_{0}$ if $g$ satisfies (2.45) and (2.46) (or (2.93) and (2.94)). Then it is sufficient to prove that $u \in\left(H^{s}(\Omega) \cap H_{\Gamma_{1}}^{1}(\Omega)\right)^{n}$ for some $s>3 / 2$ if $(u, v, \theta) \in D(\Lambda)$. For this, let $f=A u+B v$ and $h=-m \cdot \nu g(v)$. Then, for any $w \in\left(H_{\Gamma_{1}}^{1}(\Omega)\right)^{n}$, we have

$$
\begin{aligned}
\langle f, w\rangle= & \int_{\Omega}(\mu \nabla u \cdot \nabla w+(\lambda+\mu) \operatorname{div} u \operatorname{div} w) d x \\
& +\int_{\Gamma_{2}} a m \cdot \nu u \cdot w d \Gamma+\int_{\Gamma_{2}} m \cdot \nu g(v) \cdot w d \Gamma .
\end{aligned}
$$


This shows that $u$ is a weak solution of the following elliptic problem:

$$
\begin{cases}-\mu \Delta u-(\lambda+\mu) \nabla \operatorname{div} u=f & \text { in } \Omega, \\ u=0 & \text { on } \Gamma_{1}, \\ \mu \frac{\partial u}{\partial \nu}+(\lambda+\mu) \nu \operatorname{div} u+a m \cdot \nu u=h & \text { on } \Gamma_{2} .\end{cases}
$$

By assumption, we have

$$
f=A u+B v \in\left(L^{2}(\Omega)\right)^{n} .
$$

We further prove that

$$
h \in\left(H^{\sigma}(\Gamma)\right)^{n}
$$

for some $0<\sigma<q / 2$. For this, we let

$$
\begin{aligned}
S_{1} & =\{(x, y) \in \Gamma \times \Gamma:|x-y| \leq 1\}, \\
S_{11} & =\left\{(x, y) \in S_{1}:|v(x)-v(y)| \leq 1\right\}, \\
S_{12} & =\left\{(x, y) \in S_{1}:|v(x)-v(y)| \geq 1\right\} .
\end{aligned}
$$

To prove (3.43), by definition (see [15, p. 20]), it suffices to prove

$$
\iint_{\Gamma \times \Gamma} \frac{|g(v(x))-g(v(y))|^{2}}{|x-y|^{n-1+2 \sigma}} d \Gamma(x) d \Gamma(y)<\infty .
$$

The integral over $\Gamma \times \Gamma-S_{1}$ is bounded above by

$$
c \iint_{\Gamma \times \Gamma}|g(v(x))-g(v(y))|^{2} d \Gamma(x) d \Gamma(y) \leq c \int_{\Gamma}|g(v(x))|^{2} d \Gamma(x)<\infty,
$$

since $g(v(x)) \in L^{2}(\Gamma)$ which is true because $v \in\left(H^{1 / 2}(\Gamma)\right)^{n}$ and $g$ satisfies (2.45) and (2.46) (or (2.93) and (2.94)). Therefore, it suffices to prove

$$
\begin{aligned}
I= & \iint_{S_{1}} \frac{|g(v(x))-g(v(y))|^{2}}{|x-y|^{n-1+2 \sigma}} d \Gamma(x) d \Gamma(y) \\
= & \iint_{S_{11}} \frac{|g(v(x))-g(v(y))|^{2}}{|x-y|^{n-1+2 \sigma}} d \Gamma(x) d \Gamma(y) \\
& +\iint_{S_{12}} \frac{|g(v(x))-g(v(y))|^{2}}{|x-y|^{n-1+2 \sigma}} d \Gamma(x) d \Gamma(y) \\
= & I_{1}+I_{2} \\
< & \infty
\end{aligned}
$$

By (2.45) and Hölder's inequality, we have

$$
\begin{aligned}
I_{1} \leq & \iint_{S_{11}} \frac{|v(x)-v(y)|^{2 q}}{|x-y|^{n-1+2 \sigma}} d \Gamma(x) d \Gamma(y) \\
\leq & \left.\left(\iint_{S_{11}} \frac{|v(x)-v(y)|^{2}}{|x-y|^{2}} d \Gamma(x) d \Gamma(y)\right)\right)^{q} \\
& \times\left(\iint_{S_{1}} \frac{1}{|x-y|^{(n-1+2 \sigma-n q) /(1-q)}} d \Gamma(x) d \Gamma(y)\right)^{1-q} .
\end{aligned}
$$


Since $v \in\left(H_{\Gamma_{1}}^{1}(\Omega)\right)^{n}$, by the trace theorem, we have $v \in\left(H^{1 / 2}(\Gamma)\right)^{n}$, and then we have

$$
\iint_{S_{1}} \frac{|v(x)-v(y)|^{2}}{|x-y|^{n}} d \Gamma(x) d \Gamma(y)<\infty .
$$

In addition, since for $0<2 \sigma<q$

$$
\frac{n-1+2 \sigma-n q}{1-q}=n+\frac{2 \sigma-1}{1-q}<n-1,
$$

we have

$$
\iint_{S_{1}} \frac{1}{|x-y|^{(n-1+2 \sigma-n q) /(1-q)}} d \Gamma(x) d \Gamma(y)<\infty .
$$

It therefore follows that $I_{1}<\infty$. On the other hand, by (2.46) and (3.51), we have

$$
\begin{aligned}
I_{2} & \leq \iint_{S_{12}} \frac{|v(x)-v(y)|^{2}}{|x-y|^{n-1+2 \sigma}} d \Gamma(x) d \Gamma(y) \\
& \leq \iint_{S_{12}} \frac{|v(x)-v(y)|^{2}}{|x-y|^{n}} d \Gamma(x) d \Gamma(y) \\
& <\infty .
\end{aligned}
$$

Thus we have proved (3.49). In a similar way, we can show that $I<\infty$ if (2.93) holds.

Finally, we prove that

$$
u \in\left(H^{s}(\Omega) \cap H_{\Gamma_{1}}^{1}(\Omega)\right)^{n}
$$

with

$$
s=\frac{3}{2}+\sigma>\frac{3}{2}
$$

Let $\nu=\left(\nu_{1}, \nu_{2}, \ldots, \nu_{n}\right)$ denote the unit normal on $\Gamma$ directed to the exterior of $\Omega$ and consider the following system:

$$
\sum_{j=1}^{n}\left[(\lambda+\mu) \nu_{i} \nu_{j}+\delta_{i j} \mu\right] \xi_{j}=h_{i}, \quad i=1, \ldots, n,
$$

where $\delta_{i j}$ denotes the Kronecker symbol, i.e.,

$$
\delta_{i j}= \begin{cases}1, & i=j \\ 0, & i \neq j\end{cases}
$$

It is easy to see that the system has a solution $\left.\xi=\left(\xi_{1}, \ldots, \xi_{n}\right) \in\left(H^{\sigma}(\Gamma)\right)^{n}\right)$ for $h \in$ $\left(H^{\sigma}(\Gamma)\right)^{n}$. By the trace theorem (see, e.g., [36, p. 39, Theorem 8.3]), there exists $\varphi \in$ $\left(H^{s}(\Omega) \cap H_{\Gamma_{1}}^{1}(\Omega)\right)^{n}$ such that

$$
\frac{\partial \varphi}{\partial \nu}=\xi, \varphi=0 \quad \text { on } \Gamma
$$


Let $\left\{\tau^{k}(x)\right\}_{k=1}^{n-1}$ be a tangential vector field such that $\left\{\nu(x), \tau^{1}(x), \ldots, \tau^{n-1}(x)\right\}$ forms an orthonormal basis in $\mathbf{R}^{n}$ for almost all $x \in \Gamma$. Hence, there exist $\gamma^{k, j}(j=1,2, \ldots, n ; k=$ $1,2, \ldots, n-1)$ depending on $\left\{\nu(x), \tau^{1}(x), \ldots, \tau^{n-1}(x)\right\}$ such that

$$
\begin{aligned}
\frac{\partial \varphi_{j}}{\partial x_{j}} & =\nu_{j} \frac{\partial \varphi_{j}}{\partial \nu}+\sum_{k=1}^{n-1} \gamma^{k, j} \frac{\partial \varphi_{j}}{\partial \tau^{k}} \\
& =\nu_{j} \frac{\partial \varphi_{j}}{\partial \nu} \\
& =\nu_{j} \xi_{j} \quad \text { on } \Gamma, j=1,2, \ldots, n .
\end{aligned}
$$

It therefore follows from (3.57) that

$$
\mu \frac{\partial \varphi}{\partial \nu}+(\lambda+\mu) \nu \operatorname{div} \varphi+a m \cdot \nu \varphi=h \quad \text { on } \Gamma_{2}
$$

Setting

$$
\psi=u-\varphi
$$

then $\psi$ satisfies

$$
\begin{cases}-\mu \Delta \psi-(\lambda+\mu) \nabla \operatorname{div} \psi=F & \text { in } \Omega \\ \psi=0 & \text { on } \Gamma_{1} \\ \mu \frac{\partial \psi}{\partial \nu}+(\lambda+\mu) \nu \operatorname{div} \psi+a m \cdot \nu \psi=0 & \text { on } \Gamma_{2}\end{cases}
$$

where

$$
F=f-\mu \Delta \varphi-(\lambda+\mu) \nabla \operatorname{div} \varphi \in\left(H^{s-2}(\Omega)\right)^{n} .
$$

Thus, problem (3.41) is equivalent to (3.63). By the classical variational methods (see, e.g., [13]), for every $F \in\left(\left(H_{\Gamma_{1}}^{1}(\Omega)^{\prime}\right)^{n}\right.$, problem (3.63) has a unique weak solution $\psi \in$ $\left(H_{\Gamma 1}^{1}(\Omega)\right)^{n}$ in the sense of distribution

$$
\begin{aligned}
& \int_{\Omega}(\mu\nabla \psi \cdot \nabla \phi+(\lambda+\mu) \operatorname{div} \psi \operatorname{div} \phi) d x+\int_{\Gamma_{2}} a m \cdot \nu \psi \cdot \phi d \Gamma \\
&=\int_{\Omega} F \cdot \phi d x \quad \forall \phi \in\left(H_{\Gamma_{1}}^{1}(\Omega)\right)^{n} .
\end{aligned}
$$

Moreover, by the classical Nirenberg's translation method (see, e.g., [2, p. 107, Lemma 9.2] or [36, p. 124]), we prove that, if $F \in\left(L^{2}(\Omega)\right)^{n}$, then $\psi \in\left(H^{2}(\Omega) \cap H_{\Gamma_{1}}^{1}(\Omega)\right)^{n}$. Our proof is essentially the same as that of Lemma 9.2 of $[2$, p. 107]. Since the regularity is local property, it suffices to prove that, for any $x \in \bar{\Omega}$, there exists a neighborhood $O(x)$ 
such that $\psi \in x \in \Omega$ is $\psi \in\left(H^{2}(O(x) \cap \Omega)\right)^{n}$. We consider the case $x \in \Gamma$ since the case $x \in \Omega$ is much easier. For simplicity, we may as well assume that $x=0 \in \Gamma_{2}$ and that the boundary is flat with the normal oriented in the direction $x_{n}$ since the general case can be transformed into the special case by a mapping of class $C^{2}$ (see Remark 3.4 below). Therefore, there exists a hemisphere $G_{R}=\left\{x:|x|<R, x_{n}>0\right\}$ such that $G_{R} \subset \Omega$ and $\Gamma_{2 G}=\left\{x \in \bar{G}_{R}: x_{n}=0\right\} \subset \Gamma_{2}$. Let $0<R^{\prime}<R$ and $R^{\prime \prime}=\frac{1}{2}\left(R^{\prime}+R\right)$, and let $\zeta$ denote a real function that is infinitely differentiable on $\mathbf{R}^{n}$ and such that $\zeta=1$ on $G_{R^{\prime}}$ and $\zeta=0$ outside $G_{R^{\prime \prime}}$. Note that $\zeta$ need not vanish on the flat part $\Gamma_{2 G}$ of the boundary of $G_{R}$. Let $C_{0}^{\infty}\left(G_{R} \cup \Gamma_{2 G}\right)$ denote the function space of all infinitely differentiable functions with support in $G_{R} \cup \Gamma_{2 G}$. By (3.65), we have for any $\phi \in\left(C_{0}^{\infty}\left(G_{R} \cup \Gamma_{2 G}\right)\right)^{n}$ that

$$
\int_{G_{R}}(\mu \nabla \psi \cdot \nabla \phi+(\lambda+\mu) \operatorname{div} \psi \operatorname{div} \phi) d x+\int_{\Gamma_{2 G}} a m \cdot \nu \psi \cdot \phi d \Gamma=\int_{G_{R}} F \cdot \phi d x
$$

Define the bilinear form $B(\psi, \phi)$ by

$$
\begin{aligned}
B(\psi, \phi)= & \int_{G_{R}}(\mu \nabla \psi \cdot \nabla \phi+(\lambda+\mu) \operatorname{div} \psi \operatorname{div} \phi) d x \\
& +\int_{\Gamma_{2 G}} a m \cdot \nu \psi \cdot \phi d \Gamma
\end{aligned}
$$

Then we have

$$
|B(\psi, \phi)| \leq\|F\|\|\phi\| \quad \forall \phi \in\left(C_{0}^{\infty}\left(G_{R} \cup \Gamma_{2 G}\right)\right)^{n}
$$

For a real number, we define the difference operator $\delta_{h}^{i}$ by

$$
\delta_{h}^{i} u=h^{-1}\left[u\left(x_{1}, \ldots, x_{i}+h, \ldots, x_{n}\right)-u\left(x_{1}, \ldots, x_{i}, \ldots, x_{n}\right)\right], \quad i=1,2, \ldots, n
$$

We now want to estimate the difference quotients $\delta_{h}^{i}(\zeta \psi)$ for $i=1,2, \ldots, n$. Since 


$$
\begin{aligned}
& B\left(\delta_{h}^{i}(\zeta \psi, \phi)=\int_{G_{R}}\left(\mu \nabla\left(\delta_{h}^{i}(\zeta \psi)\right) \cdot \nabla \phi+(\lambda+\mu) \operatorname{div}\left(\delta_{h}^{i}(\zeta \psi)\right) \operatorname{div} \phi\right) d x\right. \\
& +\int_{\Gamma_{2 G}} a m \cdot \nu\left(\delta_{h}^{i}(\zeta \psi)\right) \cdot \phi d \Gamma \\
& =\int_{G_{R}}\left(\mu \delta_{h}^{i}(\nabla(\zeta \psi)) \cdot \nabla \phi+(\lambda+\mu) \delta_{h}^{i}(\operatorname{div}(\zeta \psi)) \operatorname{div} \phi\right) d x \\
& +\int_{2 G} a m \cdot \nu\left(\delta_{h}^{i}(\zeta \psi)\right) \cdot \phi d \Gamma \\
& =\int_{G_{R}}\left(\mu \delta_{h}^{i}\left(\psi_{j} \nabla \zeta+\zeta \nabla \psi_{j}\right) \cdot \nabla \phi_{j}+(\lambda+\mu) \delta_{h}^{i}(\nabla \zeta \cdot \psi+\zeta \operatorname{div} \psi) \operatorname{div} \phi\right) d x \\
& +\int_{\Gamma_{2 G}} a m \cdot \nu\left(\delta_{h}^{i}(\zeta \psi)\right) \cdot \phi d \Gamma \\
& =\mu \int_{G_{R}}\left(\delta_{h}^{i}\left(\psi_{j} \nabla \zeta\right) \cdot \nabla \phi_{j}+\zeta \nabla \psi_{j} \cdot \delta_{-h}^{i}\left(\nabla \phi_{j}\right)\right) d x \\
& \left.+(\lambda+\mu) \int_{G_{R}}\left(\delta_{h}^{i}(\nabla \zeta \cdot \psi) \operatorname{div} \phi+\zeta \operatorname{div} \psi\right) \delta_{-h}^{i}(\operatorname{div} \phi)\right) d x \\
& +\int_{\Gamma_{2 G}} a m \cdot \nu \zeta \psi \cdot \delta_{-h}^{i}(\phi) d \Gamma \\
& =\mu \int_{G_{R}}\left(\delta_{h}^{i}\left(\psi_{j} \nabla \zeta\right) \cdot \nabla \phi_{j}+\nabla \psi_{j} \cdot \nabla\left(\zeta \delta_{-h}^{i} \phi_{j}\right)-\nabla \psi_{j} \cdot \nabla \zeta \delta_{-h}^{i} \phi_{j}\right) d x \\
& +(\lambda+\mu) \int_{G_{R}}\left(\delta_{h}^{i}(\nabla \zeta \cdot \psi) \operatorname{div} \phi+\operatorname{div} \psi \operatorname{div}\left(\zeta \delta_{-h}^{i} \phi\right)-\operatorname{div} \psi \nabla \zeta \cdot \delta_{-h}^{i} \phi\right) d x \\
& +\int_{\Gamma_{2 G}} a m \cdot \nu \psi \cdot\left(\zeta \delta_{-h}^{i} \phi\right) d \Gamma \\
& =B\left(\psi, \zeta \delta_{-h}^{i} \phi\right)+\mu \int_{G_{R}}\left(\delta_{h}^{i}\left(\psi_{j} \nabla \zeta\right) \cdot \nabla \phi_{j}-\nabla \psi_{j} \cdot \nabla \zeta \delta_{-h}^{i} \phi_{j}\right) d x \\
& +(\lambda+\mu) \int_{G_{R}}\left(\delta_{h}^{i}(\nabla \zeta \cdot \psi) \operatorname{div} \phi-\operatorname{div} \psi \nabla \zeta \cdot \delta_{-h}^{i} \phi\right) d x
\end{aligned}
$$

It therefore follows from (3.68) that

$$
\begin{aligned}
\left|B\left(\delta_{h}^{i}(\zeta \psi), \phi\right)\right| & \leq\|F\|\left\|\zeta \delta_{-h}^{i} \phi\right\|+C\|\phi\|_{\left(H^{1}\left(G_{R}\right)\right)^{n}}\|\psi\|_{\left(H^{1}\left(G_{R}\right)\right)^{n}} \\
& \leq C\left(\|F\|+\|\psi\|_{\left.\left(H^{1}\left(G_{R}\right)\right)^{n}\right)}\|\phi\|_{\left(H^{1}\left(G_{R}\right)\right)^{n}}\right.
\end{aligned}
$$

Let $\left(H_{\Gamma_{2 G}}^{1}\left(G_{R}\right)\right)^{n}$ be the completion of $\left(C_{0}^{\infty}\left(G_{R} \cup \Gamma_{2 G}\right)\right)^{n}$ in $\left(H^{1}\left(G_{R}\right)\right)^{n}$. Then by a density argument, we obtain for any $\phi \in\left(H_{\Gamma_{2 G}}^{1}\left(G_{R}\right)\right)^{n}$

$$
\left|B\left(\delta_{h}^{i}(\zeta \psi), \phi\right)\right| \leq C\left(\|F\|+\|\psi\|_{\left.\left(H^{1}\left(G_{R}\right)\right)^{n}\right)}\|\phi\|_{\left(H^{1}\left(G_{R}\right)\right)^{n}}\right.
$$

Since $\delta_{h}^{i}(\zeta \psi) \in\left(H_{\Gamma_{2 G}}^{1}\left(G_{R}\right)\right)^{n}$ if $h$ is small enough, we deduce

$$
\left|B\left(\delta_{h}^{i}(\zeta \psi), \delta_{h}^{i}(\zeta \psi)\right)\right| \leq C\left(\|F\|+\|\psi\|_{\left(H^{1}\left(G_{R}\right)\right)^{n}}\left\|\delta_{h}^{i}(\zeta \psi)\right\|_{\left.H^{1}\left(G_{R}\right)\right)^{n}}\right.
$$


On the other hand, it is clear that

$$
\left|B\left(\delta_{h}^{i}(\zeta \psi), \delta_{h}^{i}(\zeta \psi)\right)\right| \geq C\left\|\delta_{h}^{i}(\zeta \psi)\right\|_{\left(H^{1}\left(G_{R}\right)\right)^{n}}^{2}
$$

Hence it follows from (3.72) that

$$
\left\|\delta_{h}^{i}(\zeta \psi)\right\|_{\left(H^{1}\left(G_{R}\right)\right)^{n}} \leq C\left(\|F\|+\|\psi\|_{\left.\left(H^{1}\left(G_{R}\right)\right)^{n}\right)} .\right.
$$

Since $\zeta=1$ on $G_{R^{\prime}}$, by Theorem 3.16 of $\left[2\right.$, p. 45], we deduce that $\frac{\partial \psi_{i}}{\partial x_{j}} \in\left(H^{1}\left(G_{R^{\prime}}\right)\right)^{n}$ for all $i=1, \ldots, n, j=1, \ldots, n-1$. It remains to show that $\frac{\partial^{2} \psi}{\partial x_{n}^{2}} \in\left(L^{2}\left(G_{R^{\prime}}\right)\right)^{n}$. To do this we have to distinguish the components $\psi_{i}$ for $i=1, \ldots, n-1$ and for $i=n$. In what concerns $i=1, \ldots, n-1$, we have

$$
-\mu \frac{\partial^{2} \psi_{i}}{\partial x_{n}^{2}}=\mu \Delta^{\prime} \psi_{i}+(\lambda+\mu) \frac{\partial}{\partial x_{i}}(\operatorname{div} \psi)+F_{i} \in L^{2}\left(G_{R^{\prime}}\right),
$$

while

$$
-(\lambda+2 \mu) \frac{\partial^{2} \psi_{n}}{\partial x_{n}^{2}}=\mu \Delta^{\prime} \psi_{n}+(\lambda+\mu) \frac{\partial}{\partial x_{n}}\left(\frac{\partial \psi_{1}}{\partial x_{1}}+\cdots+\frac{\partial \psi_{n-1}}{\partial x_{n-1}}\right)+F_{n} \in L^{2}\left(G_{R^{\prime}}\right)
$$

where $\Delta^{\prime}=\frac{\partial^{2}}{\partial x_{1}^{2}}+\cdots+\frac{\partial^{2}}{\partial x_{n-1}^{2}}$.

By interpolation (see, e.g., [36, p. 29, Theorem 6.2]) for $F \in\left(H^{s-2}(\Omega)\right)^{n}$ we have $\psi \in\left(H^{s}(\Omega) \cap H_{\Gamma_{1}}^{1}(\Omega)\right)^{n}$, and then $u \in\left(H^{s}(\Omega) \cap H_{\Gamma_{1}}^{1}(\Omega)\right)^{n}$. This completes the proof.

REMARK 3.4. Let us briefly explain how to deal with the case where the boundary is not flat by a flattening procedure. Suppose that $x^{0} \in \Gamma_{2}$ and the boundary of $\Omega$ near $x^{0}$ can be expressed by

$$
\Phi\left(x_{1}, x_{2}, \ldots, x_{n}\right)=0
$$

Let

$$
\begin{aligned}
y_{i} & =y_{i}\left(x_{1}, x_{2}, \ldots, x_{n}\right)=x_{i}, \quad i=1, \ldots, n-1 \\
y_{n} & =y_{n}\left(x_{1}, x_{2}, \ldots, x_{n}\right)=\Phi\left(x_{1}, x_{2}, \ldots, x_{n}\right)
\end{aligned}
$$

be the invertible mapping of class $C^{2}$ that transforms $\Omega$ into $\tilde{\Omega}$. We denote by $x=x(y)$ the inverted mapping of the above mapping $y=y(x)$ and set

$$
\tilde{\psi}(y)=\psi(x(y))
$$

By calculations, we obtain

$$
\begin{aligned}
\frac{\partial \psi_{i}}{\partial x_{j}} & =\sum_{k=1}^{n} \frac{\partial \tilde{\psi}_{i}}{\partial y_{k}} \frac{\partial y_{k}}{\partial x_{j}} \\
\frac{\partial^{2} \psi_{i}}{\partial x_{j}^{2}} & =\sum_{k, l=1}^{n} \frac{\partial^{2} \tilde{\psi}_{i}}{\partial y_{k} \partial y_{l}} \frac{\partial y_{k}}{\partial x_{j}} \frac{\partial y_{l}}{\partial x_{j}}+\sum_{k=1}^{n} \frac{\partial \tilde{\psi}_{i}}{\partial y_{k}} \frac{\partial^{2} y_{k}}{\partial x_{j}^{2}}
\end{aligned}
$$


Therefore, the first equation of (3.63) is transformed into

$$
\begin{aligned}
\tilde{F}_{i}(y) & =-\mu\left(\sum_{j, k, l=1}^{n} \frac{\partial^{2} \tilde{\psi}_{i}}{\partial y_{k} \partial y_{l}} \frac{\partial y_{k}}{\partial x_{j}} \frac{\partial y_{l}}{\partial x_{j}}+\sum_{j, k=1}^{n} \frac{\partial \tilde{\psi}_{i}}{\partial y_{k}} \frac{\partial^{2} y_{k}}{\partial x_{j}^{2}}\right) \\
& -(\lambda+\mu)\left(\sum_{j, k, l=1}^{n} \frac{\partial^{2} \tilde{\psi}_{j}}{\partial y_{k} \partial y_{l}} \frac{\partial y_{k}}{\partial x_{j}} \frac{\partial y_{l}}{\partial x_{i}}+\sum_{j, k=1}^{n} \frac{\partial \tilde{\psi}_{j}}{\partial y_{k}} \frac{\partial^{2} y_{k}}{\partial x_{j} \partial x_{i}}\right), \quad i=1, \ldots, n
\end{aligned}
$$

where $\tilde{F}_{i}(y)=F_{i}(x(y))$. Moreover, since the unit normal $\nu(x)$ on the boundary near $x^{0}$ is equal to

$$
\nu(x)=\frac{\nabla y_{n}}{\left|\nabla y_{n}\right|}
$$

we have

$$
\begin{aligned}
\frac{\partial \psi_{i}}{\partial \nu} & =\sum_{j=1}^{n} \frac{\partial \psi_{i}}{\partial x_{j}} \nu_{j}=\sum_{j, k=1}^{n} \frac{\partial \tilde{\psi}_{i}}{\partial y_{k}} \frac{\partial y_{k}}{\partial x_{j}} \frac{\partial y_{n}}{\partial x_{j}}\left|\nabla y_{n}\right|^{-1} \\
\nu_{i} \operatorname{div} \psi & =\sum_{j=1}^{n} \frac{\partial \psi_{j}}{\partial x_{j}} \nu_{i}=\sum_{j, k=1}^{n} \frac{\partial \tilde{\psi}_{j}}{\partial y_{k}} \frac{\partial y_{k}}{\partial x_{j}} \frac{\partial y_{n}}{\partial x_{i}}\left|\nabla y_{n}\right|^{-1}
\end{aligned}
$$

Hence, the boundary condition of (3.63) is transformed into the following form:

$$
\mu \sum_{j, k=1}^{n} \frac{\partial \tilde{\psi}_{i}}{\partial y_{k}} \frac{\partial y_{k}}{\partial x_{j}} \frac{\partial y_{n}}{\partial x_{j}}+(\lambda+\mu) \sum_{j, k=1}^{n} \frac{\partial \tilde{\psi}_{j}}{\partial y_{k}} \frac{\partial y_{k}}{\partial x_{j}} \frac{\partial y_{n}}{\partial x_{i}}+\tilde{a} \tilde{\psi}_{i}=0, \quad i=1, \ldots, n
$$

where $\tilde{a}=\tilde{a}(y) \geq 0$. Since the already known $H^{1}$-regularity guarantees that the firstorder derivative terms of the unknown $\tilde{\psi}$ in (3.78) are in $L^{2}$, they may be put on the right-hand side and it is then sufficient to keep the second-order terms on the left-hand side. Therefore, since $\nu(y)=(0, \ldots, 0,1)$ on the boundary of $\tilde{\Omega}$ near $y^{0}=y\left(x^{0}\right)$, it follows that the bilinear form corresponding to equation (3.78) and boundary condition $(3.81)$ is

$$
\begin{aligned}
\tilde{B}(\tilde{\psi}, \tilde{\phi})= & \mu \int_{\tilde{G}_{R}} \sum_{i, j, k, l=1}^{n} \frac{\partial \tilde{\psi}_{i}}{\partial y_{k}} \frac{\partial \tilde{\phi}_{i}}{\partial y_{l}} \frac{\partial y_{k}}{\partial x_{j}} \frac{\partial y_{l}}{\partial x_{j}} d y+(\lambda+\mu) \int_{\tilde{G}_{R}} \sum_{i, j, k, l=1}^{n} \frac{\partial \tilde{\psi}_{j}}{\partial y_{k}} \frac{\partial \tilde{\phi}_{i}}{\partial y_{l}} \frac{\partial y_{k}}{\partial x_{j}} \frac{\partial y_{l}}{\partial x_{i}} d y \\
& +\int_{\tilde{\Gamma}_{2 G}} \tilde{\alpha} \tilde{\psi} \cdot \tilde{\phi} d \Gamma
\end{aligned}
$$

where $\tilde{G}_{R}$ and $\tilde{\Gamma}_{2 G}$ denote the same subsets in the $y$-space as $G_{R}$ and $\Gamma_{2 G}$. It is clear that the bilinear $\tilde{B}(\tilde{\psi}, \tilde{\phi})$ has the same properties as $B(\psi, \phi)$. Therefore, the above proof of regularity is valid for the general case (see, e.g., [8]).

By the classical theory of nonlinear semigroups (see [6, Chap. 3]), we have

Theorem 3.5. Let $\Gamma_{1}$ and $\Gamma_{2}$ be given by (2.1) and (2.2). Let (2.16) and (2.43) hold. Suppose that the function $g$ satisfies $(2.44),(2.49)$ (or $(2.92),(2.97)$ ) and (3.4). Then we have 
(i) For every initial condition $\left(u^{0}, u^{1}, \theta^{0}\right) \in \mathcal{H}$, problem (2.7) has a unique mild solution satisfying

$$
\left(u, u^{\prime}, \theta\right) \in C([0, \infty) ; \mathcal{H})
$$

Moreover, if $(u, \theta)$ and $(v, \xi)$ are two solutions corresponding to initial states $\left(u^{0}, u^{1}, \theta^{0}\right)$ and $\left(v^{0}, v^{1}, \xi^{0}\right)$, respectively, then we have for every $t \in[0, \infty)$

$$
\left.\| u(t), u^{\prime}(t), \theta(t)\right)-\left(v(t), v^{\prime}(t), \xi(t)\right)\left\|_{\mathcal{H}} \leq\right\|\left(u^{0}, u^{1}, \theta^{0}\right)-\left(v^{0}, v^{1}, \xi^{0}\right) \|_{\mathcal{H}} .
$$

(ii) Further, if $g$ satisfies (2.45) and (2.46) (or (2.93) and (2.94)), then for every initial condition $\left(u^{0}, u^{1}, \theta^{0}\right) \in D(\Lambda)$, problem (2.7) has a unique classical solution satisfying

$$
\begin{aligned}
u & \in L^{\infty}\left([0, \infty) ;\left(H^{s}(\Omega) \cap H_{\Gamma_{1}}^{1}(\Omega)\right)^{n}\right), \\
u^{\prime} & \in L^{\infty}\left([0, \infty) ;\left(H_{\Gamma_{1}}^{1}(\Omega)\right)^{n}\right), \\
u^{\prime \prime} & \in L^{\infty}\left([0, \infty) ;\left(L^{2}(\Omega)\right)^{n}\right), \\
\theta & \in L^{\infty}\left([0, \infty) ; H^{2}(\Omega) \cap H_{0}^{1}(\Omega)\right), \\
\theta^{\prime} & \in L^{\infty}\left([0, \infty) ; L^{2}(\Omega)\right),
\end{aligned}
$$

for some $s>3 / 2$.

4. Proofs of the main results. In this section, we borrow the Lyapunov method to prove our main results.

Let $u, \theta$ be the solution of (2.7) and $\varphi$ the solution of (2.27) corresponding to the present solution $u$ of (2.7) (note that $\varphi$ depends on $t$ as $u$ does). Let $\delta$ be any positive number. We define a functional $V$ on $\mathcal{H}$ by

$$
\begin{gathered}
F(t)=F(u, \theta, t)=\int_{\Omega}\left[2 u_{i}^{\prime} m \cdot \nabla u_{i}+(n-1) u_{i}^{\prime} u_{i}+C u_{i}^{\prime} \varphi_{i}\right] d x \\
V(t)=V(u, \theta, t)=E(t)+\delta[E(t)]^{\sigma} F(t),
\end{gathered}
$$

where $C$ and $\sigma$ are nonnegative constants that will be determined later. Here we have used the summation convention for repeated indices. Thus, equation (4.1) means that

$$
F(t)=\sum_{i=1}^{n} \int_{\Omega}\left[2 u_{i}^{\prime} m \cdot \nabla u_{i}+(n-1) u_{i}^{\prime} u_{i}+C \varphi_{i} u_{i}^{\prime}\right] d x
$$

Evidently, the functional $V$ is actually a generalized energy functional which is closely related to the energy functional $E(t)$. Such similar functionals were constructed for the wave equation (see $[10,25,51]$ ), thermoelastic plate models (see $[42,43]$ ), viscoelasticity (see $[34,35]$ ) and thermoviscoelasticity (see [39]).

We will see below that the term $C u_{i}^{\prime} \varphi_{i}$ plays a key role in dealing with the case where the potential $a$ is large.

We are going to show that $V$ satisfies

$$
V^{\prime}(t) \leq-c V^{\sigma+1}(t),
$$

where $c$ is a positive constant. Then, by solving differential inequality (4.4), Theorems $2.2,2.3$ and 2.5 are proved.

We first show that, by choosing $\delta$ sufficiently small, $V$ and $E$ are equivalent. 
Lemma 4.1. Let $\Gamma_{1}$ and $\Gamma_{2}$ be given by (2.1) and (2.2), respectively. Suppose that (2.16) holds. Let the function $g \in\left(C\left(\mathbf{R}^{n}\right)\right)^{n}$ satisfy (2.44), (2.49) (or (2.92), (2.97)) and (3.4). Then we have

$$
\left.\left.\left(1-\delta C_{1}^{\prime \prime} E^{\sigma}(t)\right)\right) E(t) \leq V(t) \leq\left(1+\delta C_{1}^{\prime \prime} E^{\sigma}(t)\right)\right) E(t),
$$

for all solutions $u, \theta$ of $(2.7)$, where the positive constant $C_{1}^{\prime \prime}$ is given by

$$
C_{1}^{\prime \prime}=\frac{2 R_{0}}{\sqrt{\mu}}+(n-1) \lambda_{0}+C k_{1} \gamma
$$

Proof. It is easy to see that

$$
\left|2 \int_{\Omega} u_{i}^{\prime} m \cdot \nabla u_{i} d x\right| \leq \frac{R_{0}}{\sqrt{\mu}}\left[\left\|u_{i}^{\prime}(t)\right\|^{2}+\mu\left\|\nabla u_{i}(t)\right\|^{2}\right] \leq \frac{2 R_{0}}{\sqrt{\mu}} E(t) .
$$

Using (2.25), we obtain

$$
\left|\int_{\Omega} u_{i}^{\prime} u_{i} d x\right| \leq \frac{\lambda_{0}}{2}\left[\left\|u^{\prime}(t)\right\|^{2}+\|u(t)\|_{\left(H_{\Gamma_{1}}^{1}(\Omega)\right)^{n}}^{2}\right] \leq \lambda_{0} E(t) .
$$

Using (2.28) and (2.24), we have

$$
\begin{aligned}
\left|\int_{\Omega} u_{i}^{\prime} \varphi_{i} d x\right| & =\frac{k_{1} \gamma}{2}\left|\int_{\Omega} 2 u_{i}^{\prime} \frac{\varphi_{i}}{k_{1} \gamma} d x\right| \\
& \leq \frac{k_{1} \gamma}{2}\left[\left\|u_{i}^{\prime}(t)\right\|^{2}+\frac{1}{k_{1}^{2} \gamma^{2}}\left\|\varphi_{i}(t)\right\|^{2}\right] \\
& \leq \frac{k_{1} \gamma}{2}\left[\left\|u_{i}^{\prime}(t)\right\|^{2}+\frac{1}{\gamma^{2}} \int_{\Gamma_{2}}\left|u_{i}\right|^{2} d \Gamma\right] \\
& \leq \frac{k_{1} \gamma}{2}\left[\left\|u^{\prime}(t)\right\|^{2}+\|u(t)\|_{\left(H_{\Gamma_{1}}^{1}(\Omega)\right)^{n}}^{2}\right] \\
& \leq k_{1} \gamma E(t) .
\end{aligned}
$$

Noting (4.6), we deduce from (4.7), (4.8) and (4.9) that

$$
|F(t)| \leq C_{1}^{\prime \prime} E(t),
$$

which implies (4.5).

We then estimate $F^{\prime}(t)$.

Lemma 4.2. Let $\Gamma_{1}$ and $\Gamma_{2}$ be given by (2.1) and (2.2), respectively, satisfying (2.43). Suppose that (2.16) holds and the function $g \in\left(C\left(\mathbf{R}^{n}\right)\right)^{n}$ satisfies (2.44), (2.45), (2.46) and (2.49) (or (2.92), (2.93), (2.94) and (2.97)). Let $u, \theta$ be a classical solution of (2.7) with $\left(u^{0}, u^{1}, \theta^{0}\right) \in D(\Lambda)$. Then we have

$$
\begin{aligned}
& F^{\prime}(t) \leq-E(t)+C_{2}^{\prime \prime}\|\nabla \theta(t)\|^{2} \\
& \quad+\int_{\Gamma_{2}}\left[\left(m \cdot \nu+\frac{C^{2} k_{1}^{2}}{4 \varepsilon}\right)\left|u^{\prime}\right|^{2}+C_{3}^{\prime \prime} m \cdot \nu\left|g\left(u^{\prime}\right)\right|^{2}\right] d \Gamma,
\end{aligned}
$$


where

$$
\begin{aligned}
& C_{2}^{\prime \prime}=\frac{\alpha^{2} R_{0}^{2}}{\mu \varepsilon}+\frac{\alpha^{2}(n-1)^{2} \lambda_{0}^{2}}{4 \varepsilon}+\frac{C^{2} \alpha^{2} k_{1}^{2} \gamma^{2}}{4 \varepsilon}+\frac{\alpha \lambda_{1}^{2}}{\beta}, \\
& C_{3}^{\prime \prime}=\frac{2 R_{0}^{2}}{\mu}+\frac{(n-1+C)^{2} R_{0} \gamma^{2}}{4 \varepsilon},
\end{aligned}
$$

and the constant $C$ is given by

$$
C=C_{0}=\max \left\{0, \frac{2 a_{0} R_{0}^{2}}{\mu}+2-n\right\} .
$$

If, further, the function $a(x)$ satisfies $(2.50)$ or (2.51), then we can take

$$
C=0 \text {. }
$$

The constant $\varepsilon$ is given by

$$
\varepsilon= \begin{cases}1 / 8, & \text { if } C=C_{0}, \\ 1 / 8, & \text { if } C=0 \text { and } a(x) \text { satisfied (2.51), } \\ \left(1-2 K(a) R_{0} \gamma^{2}\right) / 8, & \text { if } C=0 \text { and } a(x) \text { satisfies (2.50). }\end{cases}
$$

Proof. Since $\left(u^{0}, u^{1}, \theta^{0}\right) \in D(\Lambda)$, we have $u \in L^{\infty}\left([0, \infty) ;\left(H^{s}(\Omega) \cap H_{\Gamma_{1}}^{1}(\Omega)\right)^{n}\right)$ for some $s>3 / 2$ and $\theta \in L^{\infty}\left([0, \infty) ; H^{2}(\Omega) \cap H_{0}^{1}(\Omega)\right)$. Hence, the following integrations by parts are valid.

By (4.1), we have

$$
\begin{aligned}
F^{\prime}(t)= & \int_{\Omega} 2 u_{i}^{\prime \prime} m \cdot \nabla u_{i} d x+\int_{\Omega} 2 u_{i}^{\prime} m \cdot \nabla u_{i}^{\prime} d x \\
& +\int_{\Omega}(n-1) u_{i} u_{i}^{\prime \prime} d x+(n-1)\left\|u_{i}^{\prime}\right\|^{2} \\
& +C \int_{\Omega} \varphi_{i} u_{i}^{\prime \prime} d x+C \int_{\Omega} \varphi_{i}^{\prime} u_{i}^{\prime} d x
\end{aligned}
$$

We now estimate every integral in (4.17) as follows. Since $u=0$ on $\Gamma_{1}$, we have

$$
\frac{\partial u_{i}}{\partial x_{k}}=\frac{\partial u_{i}}{\partial \nu} \nu_{k} \quad \text { on } \Gamma_{1}
$$


Thus, we obtain

$$
\begin{aligned}
& 2 \int_{\Omega} u_{i}^{\prime \prime} m \cdot \nabla u_{i} d x \\
&=2 \int_{\Omega}\left[\mu \Delta u_{i}+(\lambda+\mu) \frac{\partial}{\partial x_{i}}(\operatorname{div} u)-\alpha \frac{\partial \theta}{\partial x_{i}}\right] m \cdot \nabla u_{i} d x \\
&= \mu \int_{\Gamma}\left[2 \frac{\partial u_{i}}{\partial \nu} m \cdot \nabla u_{i}-m \cdot \nu\left|\nabla u_{i}\right|^{2}\right] d \Gamma+(n-2) \mu\left\|\nabla u_{i}\right\|^{2} \\
&+(\lambda+\mu) \int_{\Gamma}\left[2 \operatorname{div}(u) m_{k} \nu_{i} \frac{\partial u_{i}}{\partial x_{k}}-m \cdot \nu|\operatorname{div} u|^{2}\right] d \Gamma \\
&+(n-2)(\lambda+\mu)\|\operatorname{div} u\|^{2}-2 \alpha \int_{\Omega}\left(m \cdot \nabla u_{i}\right) \frac{\partial \theta}{\partial x_{i}} d x \\
&= \int_{\Gamma_{1}} m \cdot \nu\left[\mu\left|\frac{\partial u_{i}}{\partial \nu}\right|^{2}+(\lambda+\mu)|\operatorname{div} u|^{2}\right] d \Gamma \\
&+2 \int_{\Gamma_{2}}\left[\mu \frac{\partial u_{i}}{\partial \nu}+(\lambda+\mu) \nu_{i} \operatorname{div} u\right] m \cdot \nabla u_{i} d \Gamma \\
&-\int_{\Gamma_{2}} m \cdot \nu\left[\mu\left|\nabla u_{i}\right|^{2}+(\lambda+\mu)|\operatorname{div} u|^{2}\right] d \Gamma \\
&+(n-2)\left[\mu\left\|\nabla u_{i}\right\|^{2}+(\lambda+\mu)\|\operatorname{div} u\|^{2}\right] \\
&-2 \alpha \int_{\Omega}\left(m \cdot \nabla u_{i}\right) \frac{\partial \theta}{\partial x_{i}} d x .
\end{aligned}
$$

Since $m \cdot \nu \leq 0$ on $\Gamma_{1}$, we have

$$
\int_{\Gamma_{1}} m \cdot \nu\left[\mu\left|\frac{\partial u_{i}}{\partial \nu}\right|^{2}+(\lambda+\mu)|\operatorname{div} u|^{2}\right] d \Gamma \leq 0
$$

In addition, we have

$$
-2 \alpha \int_{\Omega}\left(m \cdot \nabla u_{i}\right) \frac{\partial \theta}{\partial x_{i}} d x \leq \varepsilon \mu\left\|\nabla u_{i}(t)\right\|^{2}+\frac{\alpha^{2} R_{0}^{2}}{\mu \varepsilon}\|\nabla \theta(t)\|^{2} .
$$

It therefore follows from (4.19)-(4.21) that

$$
\begin{aligned}
& 2 \int_{\Omega} u_{i}^{\prime \prime} m \cdot \nabla u_{i} d x \\
& \leq 2 \int_{\Gamma_{2}}\left[\mu \frac{\partial u_{i}}{\partial \nu}+(\lambda+\mu) \nu_{i} \operatorname{div} u\right] m \cdot \nabla u_{i} d \Gamma \\
&-\int_{\Gamma_{2}} m \cdot \nu\left[\mu\left|\nabla u_{i}\right|^{2}+(\lambda+\mu)|\operatorname{div} u|^{2}\right] d \Gamma \\
&+(n-2)\left[\mu\left\|\nabla u_{i}\right\|^{2}+(\lambda+\mu)\|\operatorname{div} u\|^{2}\right] \\
&+\varepsilon \mu\left\|\nabla u_{i}(t)\right\|^{2}+\frac{\alpha^{2} R_{0}^{2}}{\mu \varepsilon}\|\nabla \theta(t)\|^{2} .
\end{aligned}
$$

For the second integral in (4.17), we have

$$
2 \int_{\Omega} u_{i}^{\prime} m \cdot \nabla u_{i}^{\prime} d x=-n\left\|u_{i}^{\prime}(t)\right\|^{2}+\int_{\Gamma_{2}} m \cdot \nu\left|u_{i}^{\prime}\right|^{2} d \Gamma .
$$


Using (2.25), we deduce

$$
\begin{aligned}
(n-1) & \int_{\Omega} u_{i} u_{i}^{\prime \prime} d x \\
= & (n-1) \int_{\Omega} u_{i}\left[\mu \Delta u_{i}+(\lambda+\mu) \frac{\partial}{\partial x_{i}}(\operatorname{div} u)-\alpha \frac{\partial \theta}{\partial x_{i}}\right] d x \\
= & (n-1) \int_{\Gamma_{2}}\left[\mu \frac{\partial u_{i}}{\partial \nu}+(\lambda+\mu) \nu_{i} \operatorname{div} u\right] u_{i} d \Gamma \\
& -(n-1)\left[\mu\left\|\nabla u_{i}(t)\right\|^{2}+(\lambda+\mu)\|\operatorname{div} u(t)\|^{2}\right] \\
& -(n-1) \alpha \int_{\Omega} u_{i} \frac{\partial \theta}{\partial x_{i}} d x \\
\leq & (n-1) \int_{\Gamma_{2}}\left[\mu \frac{\partial u_{i}}{\partial \nu}+(\lambda+\mu) \nu_{i} \operatorname{div} u\right] u_{i} d \Gamma \\
& -(n-1)\left[\mu\left\|\nabla u_{i}(t)\right\|^{2}+(\lambda+\mu)\|\operatorname{div} u(t)\|^{2}\right] \\
& +(n-1)^{2} \frac{\alpha^{2} \lambda_{0}^{2}}{4 \varepsilon}\|\nabla \theta(t)\|^{2}+\varepsilon\|u(t)\|_{\left(H_{\Gamma_{1}}^{1}(\Omega)\right)^{n}}^{2}
\end{aligned}
$$

By (2.28) and (2.29), we have

$$
\begin{aligned}
C \int_{\Omega} \varphi_{i} & u_{i}^{\prime \prime} d x \\
= & C \int_{\Omega} \varphi_{i}\left[\mu \Delta u_{i}+(\lambda+\mu) \frac{\partial}{\partial x_{i}}(\operatorname{div} u)-\alpha \frac{\partial \theta}{\partial x_{i}}\right] d x \\
= & C \int_{\Omega_{2}}\left[\mu \frac{\partial u_{i}}{\partial \nu}+(\lambda+\mu) \nu_{i} \operatorname{div} u\right] \varphi_{i} d \Gamma \\
& -C \int_{\Omega}\left[\mu \nabla u_{i}(t) \nabla \varphi_{i}(t)+(\lambda+\mu) \operatorname{div} u(t) \operatorname{div} \varphi(t)\right] d x \\
& -C \alpha \int_{\Omega} \varphi_{i} \frac{\partial \theta}{\partial x_{i}} d x \\
\leq & C \int_{\Gamma_{2}}\left[\mu \frac{\partial u_{i}}{\partial \nu}+(\lambda+\mu) \nu_{i} \operatorname{div} u\right] u_{i} d \Gamma \\
& +\frac{C^{2} \alpha^{2} k_{1}^{2} \gamma^{2}}{4 \varepsilon}\|\nabla \theta(t)\|^{2}+\frac{\varepsilon}{k_{1}^{2} \gamma^{2}}\left\|\varphi_{i}(t)\right\|^{2} \\
\leq & C \int_{\Gamma_{2}}\left[\alpha \frac{\partial u_{i}}{\partial \nu}+(\lambda+\mu) \nu_{i} \operatorname{div}\right] u_{i} d \Gamma \\
& +\frac{C^{2} \alpha^{2} k_{1}^{2} \gamma^{2}}{4 \varepsilon}\|\nabla \theta(t)\|^{2}+\varepsilon\|u(t)\|_{\left(H_{\Gamma_{1}}(\Omega)\right)^{n}}^{2} .
\end{aligned}
$$

Using (2.28), we have

$$
\begin{aligned}
C \int_{\Omega} \varphi_{i}^{\prime} u_{i}^{\prime} d x & \leq \frac{C^{2}}{4 \varepsilon}\left\|\varphi_{i}^{\prime}(t)\right\|^{2}+\varepsilon\left\|u_{i}^{\prime}(t)\right\|^{2} \\
& \leq \frac{C^{2} k_{1}^{2}}{4 \varepsilon} \int_{\Gamma_{2}}\left|u_{i}^{\prime}(t)\right|^{2} d \Gamma+\varepsilon\left\|u_{i}^{\prime}(t)\right\|^{2}
\end{aligned}
$$


It therefore follows from (4.17) and (4.22)-(4.26) that

$$
\begin{aligned}
F^{\prime}(t) \leq & -\left[\mu\left\|\nabla u_{i}\right\|^{2}+(\lambda+\mu)\|\operatorname{div} u\|^{2}+\left\|u_{i}^{\prime}(t)\right\|^{2}\right] \\
& +3 \varepsilon\|u(t)\|_{\left(H_{\Gamma_{1}}^{1}(\Omega)\right)^{n}}^{2}+\varepsilon\left\|u_{i}^{\prime}(t)\right\|^{2} \\
& +\left[\frac{\alpha^{2} R_{0}^{2}}{\mu \varepsilon}+\frac{(n-1)^{2} \alpha^{2} \lambda_{0}^{2}}{4 \varepsilon}+\frac{C^{2} \alpha^{2} k_{1}^{2} \gamma^{2}}{4 \varepsilon}\right]\|\nabla \theta(t)\|^{2} \\
& +2 \int_{\Gamma_{2}}\left[\mu \frac{\partial u_{i}}{\partial \nu}+(\lambda+\mu) \nu_{i} \operatorname{div} u\right] m \cdot \nabla u_{i} d \Gamma \quad\left(=I_{1}\right) \\
& -\int_{\Gamma_{2}} m \cdot \nu\left[\mu\left|\nabla u_{i}\right|^{2}+(\lambda+\mu)|\operatorname{div} u|^{2}\right] d \Gamma \quad\left(=I_{2}\right) \\
& +\int_{\Gamma_{2}}\left[m \cdot \nu+\frac{C^{2} k_{1}^{2}}{4 \varepsilon}\right]\left|u_{i}^{\prime}(t)\right|^{2} d \Gamma \quad\left(=I_{3}\right) \\
& +(n-1+C) \int_{\Gamma_{2}}\left[\mu \frac{\partial u_{i}}{\partial \nu}+(\lambda+\mu) \nu_{i} \operatorname{div} u\right] u_{i} d \Gamma \quad\left(=I_{4}\right) \\
\leq & -2 E(t)+6 \varepsilon E(t) \\
& +\left[\frac{\alpha^{2} R_{0}^{2}}{\mu \varepsilon}+\frac{(n-1)^{2} \alpha^{2} \lambda_{0}^{2}}{4 \varepsilon}+\frac{C^{2} \alpha^{2} k_{1}^{2} \gamma^{2}}{4 \varepsilon}\right]\|\nabla \theta(t)\|^{2}+\frac{\alpha}{\beta}\|\theta(t)\|^{2} \\
& +I_{1}+I_{2}+I_{3}+I_{4} \\
& +\int_{\Gamma_{2}} a m \cdot \nu\left|u_{i}\right|^{2} d \Gamma \quad\left(=I_{5}\right) .
\end{aligned}
$$

Note that we add here two terms

$$
\frac{\alpha}{\beta}\|\theta(t)\|^{2}, \quad \int_{\Gamma_{2}} a m \cdot \nu\left|u_{i}\right|^{2} d \Gamma
$$

to make up $\left[\mu\left\|\nabla u_{i}\right\|^{2}+(\lambda+\mu)\|\operatorname{div} u\|^{2}+\left\|u_{i}^{\prime}(t)\right\|^{2}\right]$ into $E(t)$. Since

$$
\begin{aligned}
I_{1} & =-2 \int_{\Gamma_{2}}\left[a m \cdot \nu u_{i}+m \cdot \nu g_{i}\left(u^{\prime}\right)\right] m \cdot \nabla u_{i} d \Gamma \\
& \leq \int_{\Gamma_{2}} m \cdot \nu\left[\frac{2 \alpha^{2} R_{0}^{2}}{\mu}\left|u_{i}\right|^{2}+\frac{2 R_{0}^{2}}{\mu}\left|g\left(u^{\prime}\right)\right|^{2}+\mu\left|\nabla u_{i}\right|^{2}\right] d \Gamma
\end{aligned}
$$

we have

$$
I_{1}+I_{2} \leq \int_{\Gamma_{2}} m \cdot \nu\left[\frac{2 a^{2} R_{0}^{2}}{\mu}\left|u_{i}\right|^{2}+\frac{2 R_{0}^{2}}{\mu}\left|g\left(u^{\prime}\right)\right|^{2}\right] d \Gamma .
$$


In addition,

$$
\begin{aligned}
I_{4}+I_{5}= & -(n-1+C) \int_{\Gamma_{2}}\left[a m \cdot \nu u_{i}+m \cdot \nu g_{i}\left(u^{\prime}\right)\right] u_{i} d \Gamma+\int_{\Gamma_{2}} a m \cdot \nu\left|u_{i}\right|^{2} d \Gamma \\
\leq & -(n-2+C) \int_{\Gamma_{2}} a m \cdot \nu\left|u_{i}\right|^{2} d \Gamma \\
& +\int_{\Gamma_{2}} m \cdot \nu\left[\frac{(n-1+C)^{2} R_{0} \gamma^{2}}{4 \varepsilon}\left|g_{i}\left(u^{\prime}\right)\right|^{2}+\frac{\varepsilon}{R_{0} \gamma^{2}}\left|u_{i}\right|^{2}\right] d \Gamma \\
\leq & -(n-2+C) \int_{\Gamma_{2}} a m \cdot \nu\left|u_{i}\right|^{2} d \Gamma \\
& +\frac{(n-1+C)^{2} R_{0} \gamma^{2}}{4 \varepsilon} \int_{\Gamma_{2}} m \cdot \nu\left|g\left(u^{\prime}\right)\right|^{2} d \Gamma+\varepsilon\|u(t)\|_{\left(H_{\Gamma_{1}}^{1}(\Omega)\right)^{n}}^{2}
\end{aligned}
$$

Noting definitions (4.12) and (4.13) of $C_{2}^{\prime \prime}$ and $C_{3}^{\prime \prime}$, it therefore follows from (4.27)-(4.31) that

$$
\begin{aligned}
F^{\prime}(t) \leq & -2 E(t)+8 \varepsilon E(t)+C_{2}^{\prime \prime}\|\nabla \theta(t)\|^{2} \\
& +\int_{\Gamma_{2}}\left[m \cdot \nu+\frac{C^{2} k_{1}^{2}}{4 \varepsilon}\right]\left|u^{\prime}\right|^{2} d \Gamma+C_{3}^{\prime \prime} \int_{\Gamma_{2}} m \cdot \nu\left|g\left(u^{\prime}\right)\right|^{2} d \Gamma \\
& \left.+\int_{\Gamma_{2}} a m \cdot \nu\left[\frac{2 a R_{0}^{2}}{\mu}-n+2-C\right)\right]\left|u_{i}\right|^{2} d \Gamma \quad(=I) .
\end{aligned}
$$

Thus, by taking $C=C_{0}$ and $\varepsilon=1 / 8$, we have $I \leq 0$, and then we deduce (4.11).

If, further, the function $a(x)$ satisfies (2.51), we can take $C=0$ and we still have $I \leq 0$. Then (4.11) follows.

Likewise, if the function $a(x)$ satisfies $(2.50)$, then we can also take $C=0$. However, in this case, since $I$ is no longer negative, we estimate $I$ as follows. Let $K(a)$ be given by (2.23). Using (2.24), we deduce

$$
\begin{aligned}
I & \leq K(a) \int_{\Gamma_{2}} m \cdot \nu|u|^{2} d \Gamma \\
& \leq K(a) R_{0} \gamma^{2}\|u(t)\|_{\left(H_{\Gamma_{1}}^{1}(\Omega)\right)^{n}}^{2} \\
& \leq 2 K(a) R_{0} \gamma^{2} E(t) .
\end{aligned}
$$

Thus, by taking $\varepsilon=\left(1-2 K(a) R_{0} \gamma^{2}\right) / 8$, we also deduce (4.11). This completes the proof of Lemma 4.2.

We are now ready to prove our main results. The method of the proof is analogous to the one developed by the second author in [51].

Proof of Theorem 2.2. We first assume that $\left(u^{0}, u^{1}, \theta^{0}\right) \in D(\Lambda)$. Then Lemma 4.2 is valid. We note that $C=0$ because the function $a(x)$ satisfies $(2.50)$ or $(2.51)$. Then the constant $C_{1}^{\prime \prime}$ in Lemma 4.1 and the constants $C_{2}^{\prime \prime}$ and $C_{3}^{\prime \prime}$ in Lemma 4.2 become $C_{1}, C_{2}, C_{3}$ 
in Theorem 2.2, respectively. By (2.42), (4.10) and Lemmas 4.1 and 4.2, we have

$$
\begin{aligned}
V^{\prime}(t)= & E^{\prime}(t)+\delta \frac{d}{d t}\left(E^{\sigma}(t) F(t)\right) \\
= & E^{\prime}(t)+\delta \sigma E^{\sigma-1}(t) E^{\prime}(t) F(t)+\delta E^{\sigma}(t) F^{\prime}(t) \\
\leq & {\left[1-\delta C_{1} \sigma E^{\sigma}(t)\right] E^{\prime}(t)-\delta E^{\sigma+1}(t) } \\
& +\delta C_{2} E^{\sigma}(t)\|\nabla \theta(t)\|^{2}+\delta E^{\sigma}(t) \int_{\Gamma_{2}} m \cdot \nu\left[\left|u^{\prime}\right|^{2}+C_{3}\left|g\left(u^{\prime}\right)\right|^{2}\right] d \Gamma \\
= & -\delta E^{\sigma+1}(t)+\left[\delta E^{\sigma}(t)\left(\frac{\sigma \alpha C_{1}}{\beta}+C_{2}\right)-\frac{\alpha}{\beta}\right]\|\nabla \theta(t)\|^{2} \\
& -\left[1-\delta C_{1} \sigma E^{\sigma}(t)\right] \int_{\Gamma_{2}} m \cdot \nu g\left(u^{\prime}\right) \cdot u^{\prime} d \Gamma \\
& +\delta E^{\sigma}(t) \int_{\Gamma_{2}} m \cdot \nu\left[\left|u^{\prime}\right|^{2}+C_{3}\left|g\left(u^{\prime}\right)\right|^{2}\right] d \Gamma .
\end{aligned}
$$

We now distinguish the cases $p=q=1$ and $p+1>2 q$.

Case I: $p=q=1$. In this case, we take $\sigma=0$ and $\delta=\delta_{1}$ (see (2.64)) in (4.34). By definitions (2.64) and (2.59) of $\delta_{1}$ and $C_{4}$, it follows from (4.34) and (2.49) that

$$
\begin{aligned}
V^{\prime}(t) \leq & -\delta_{1} E(t)-\int_{\Gamma_{2}} m \cdot \nu g\left(u^{\prime}\right) \cdot u^{\prime} d \Gamma \\
& +\delta_{1} C_{4} \int_{\Gamma_{2}} m \cdot \nu\left|u^{\prime}\right|^{2} d \Gamma \\
\leq & -\delta_{1} E(t)+\left[\delta_{1} C_{4}-k_{3}\right] \int_{\Gamma_{2}} m \cdot \nu\left|u^{\prime}\right|^{2} d \Gamma \\
\leq & -\delta_{1} E(t) \quad(\text { use }(4.5)) \\
\leq & -\frac{\delta_{1}}{1+\delta_{1} C_{1}} V(t) .
\end{aligned}
$$

Solving this differential inequality and using (4.5), we obtain

$$
\begin{aligned}
E(t) & \leq \frac{1}{1-\delta_{1} C_{1}} V(t) \\
& \leq \frac{1}{1-\delta_{1} C_{1}} V(0) e^{-\delta_{1} t /\left(1+\delta_{1} C_{1}\right)} \\
& \leq \frac{1+\delta_{1} C_{1}}{1-\delta_{1} C_{1}} E(0) e^{-\delta_{1} t /\left(1+\delta_{1} C_{1}\right)}
\end{aligned}
$$

This is (2.52).

Case II: $p+1>2 q$. We first estimate

$$
E^{\sigma}(t) \int_{\Gamma_{2} \cap\left[\left|u^{\prime}\right| \leq 1\right]} m \cdot \nu\left[\left|u^{\prime}\right|^{2}+C_{3}\left|g\left(u^{\prime}\right)\right|^{2}\right] d \Gamma .
$$

Applying Young's inequality

$$
a b \leq \frac{a^{s}}{s}+\frac{b^{\tau}}{\tau}, \quad \forall a, b \geq 0, \quad \text { and } s, \tau>0 \text { with } \frac{1}{s}+\frac{1}{\tau}=1,
$$


we have for any $b>0$

$$
\begin{aligned}
E^{\sigma} & \int_{\Gamma_{2} \cap\left[\left|u^{\prime}\right| \leq 1\right]} m \cdot \nu\left|u^{\prime}\right|^{2 q} d \Gamma \\
\leq & \frac{p+1-2 q}{p+1} b^{(p+1) /(p+1-2 q)} E^{\sigma(p+1) /(p+1-2 q)} \\
& +\frac{2 q}{(p+1) b^{(p+1) /(2 q)}}\left(\int_{\Gamma_{2} \cap\left[\left|u^{\prime}\right| \leq 1\right]} m \cdot \nu\left|u^{\prime}\right|^{2 q} \Gamma\right)^{(p+1) /(2 q)} \\
\leq & \frac{p+1-2 q}{p+1} b^{(p+1) /(p+1-2 q)} E^{\sigma(p+1) /(p+1-2 q)} \\
& +\frac{2 q}{(p+1) b^{(p+1) /(2 q)}}\left(\int_{\Gamma_{2} \cap\left[\left|u^{\prime}\right| \leq 1\right]} m \cdot \nu d \Gamma\right)^{(p+1-2 q) /(2 q)} \\
& \times \int_{\Gamma_{2} \cap\left[\left|u^{\prime}\right| \leq 1\right]} m \cdot \nu\left|u^{\prime}\right|^{p+1} d \Gamma \\
\leq & \frac{p+1-2 q}{p+1} b^{(p+1) /(p+1-2 q)} E^{\sigma(p+1) /(p+1-2 q)} \\
& +\frac{2 q\left[R_{0} \operatorname{mes}\left(\Gamma_{2}\right)\right]^{(p+1-2 q) /(2 q)}}{k_{3}(p+1) b^{(p+1) /(2 q)}} \int_{\Gamma_{2} \cap\left[\left|u^{\prime}\right| \leq 1\right]} m \cdot \nu g\left(u^{\prime}\right) \cdot u^{\prime} d \Gamma .
\end{aligned}
$$

Thus, by taking

$$
b=\left(\frac{p+1}{2 C_{4}(p+1-2 q)}\right)^{(p+1-2 q) /(p+1)}
$$

we obtain

$$
\begin{aligned}
& E^{\sigma}(t) \int_{\Gamma_{2} \cap\left[u^{\prime} \mid \leq 1\right]} m \cdot \nu\left[\left|u^{\prime}\right|^{2}+C_{3}\left|g\left(u^{\prime}\right)\right|^{2}\right] d \Gamma \\
& \text { (note that } \left.\left|u^{\prime}\right|^{2} \leq\left|u^{\prime}\right|^{2 q} \text { since } q \leq 1\right) \\
& \leq C_{4} E^{\sigma}(t) \int_{\Gamma_{2} \cap\left[\left|u^{\prime}\right| \leq 1\right]} m \cdot \nu\left|u^{\prime}\right|^{2 q} \Gamma \\
& \leq \frac{1}{2} E^{\sigma(p+1) /(p+1-2 q)} \\
& \quad+\frac{q\left(2 C_{4}\right)^{(p+1) /(2 q)}\left[R_{0} \operatorname{mes}\left(\Gamma_{2}\right)(p+1-2 q)\right]^{(p+1-2 q) /(2 q)}}{k_{3}(p+1)^{(p+1) /(2 q)}} \\
& \quad \times \int_{\Gamma_{2} \cap\left[\left|u^{\prime}\right| \leq 1\right]} m \cdot \nu g\left(u^{\prime}\right) \cdot u^{\prime} d \Gamma .
\end{aligned}
$$


It therefore follows from (4.34) and (4.41) that

$$
\begin{aligned}
& V^{\prime}(t) \leq-\delta E^{\sigma+1}(t)+\left[\delta E^{\sigma}(t)\left(\frac{\sigma \alpha C_{1}}{\beta}+C_{2}\right)-\frac{\alpha}{\beta}\right]\|\nabla \theta(t)\|^{2} \\
&+\left[\delta C_{1} \sigma E^{\sigma}(0)+\frac{\delta C_{4} E^{\sigma}(0)}{k_{3}}-1\right] \int_{\Gamma_{2} \cap\left[\left|u^{\prime}\right| \geq 1\right]} m \cdot \nu g\left(u^{\prime}\right) \cdot u^{\prime} d \Gamma \\
&+\left[\delta C_{1} \sigma E^{\sigma}(0)-1\right] \int_{\Gamma_{2} \cap\left[\left|u^{\prime}\right| \leq 1\right]} m \cdot \nu g\left(u^{\prime}\right) \cdot u^{\prime} d \Gamma \\
&+\delta E^{\sigma}(t) \int_{\Gamma_{2} \cap\left[\left|u^{\prime}\right| \leq 1\right]} m \cdot \nu\left[\left|u^{\prime}\right|^{2}+C_{3}\left|g\left(u^{\prime}\right)\right|^{2}\right] d \Gamma \\
& \leq-\delta E^{\sigma+1}(t)+\frac{\delta}{2} E^{\sigma(p+1) /(p+1-2 q)} \\
&+\left[\delta E^{\sigma}(t)\left(\frac{\sigma \alpha C_{1}}{\beta}+C_{2}\right)-\frac{\alpha}{\beta}\right]\|\nabla \theta(t)\|^{2} \\
&+\left[\delta C_{1} \sigma E^{\sigma}(0)+\frac{\delta C_{4} E^{\sigma}(0)}{k_{3}}-1\right] \int_{\Gamma_{2} \cap\left[\left|u^{\prime}\right| \geq 1\right]} m \cdot \nu g\left(u^{\prime}\right) \cdot u^{\prime} d \Gamma \\
&+\left[\delta C_{1} \sigma E^{\sigma}(0)-1\right] \int_{\Gamma_{2} \cap\left[\left|u^{\prime}\right| \leq 1\right]} m \cdot \nu g\left(u^{\prime}\right) \cdot u^{\prime} d \Gamma \\
&+\delta \frac{q\left(2 C_{4}\right)^{(p+1) /(2 q)}\left[R_{0} \operatorname{mes}\left(\Gamma_{2}\right)(p+1-2 q)\right]^{(p+1-2 q) /(2 q)}}{k_{3}(p+1)^{(p+1) /(2 q)}} \\
& \times \int_{\Gamma_{2} \cap\left[\left|u^{\prime}\right| \leq 1\right]} m \cdot \nu g\left(u^{\prime}\right) \cdot u^{\prime} d \Gamma .
\end{aligned}
$$

We now choose $\sigma$ so that

$$
\sigma+1=\frac{\sigma(p+1)}{p+1-2 q} .
$$

Then

$$
\sigma=\frac{p+1-2 q}{2 q}=\sigma_{0}
$$

By taking $\delta=\delta_{2}$ (see (2.65)) and $\sigma=\sigma_{0}$ in (4.42), we obtain

$$
V^{\prime}(t) \leq-\frac{\delta_{2}}{2} E^{\sigma_{0}+1}(t)
$$

By (4.5), we have

$$
V(t) \leq\left[1+\delta_{2} C_{1} E^{\sigma_{0}}(0)\right] E(t)
$$

It therefore follows from (4.45) that

$$
V^{\prime}(t) \leq-\frac{\delta_{2}}{2\left[1+\delta_{2} C_{1} E^{\sigma_{0}}(0)\right]^{\sigma_{0}}} V^{\sigma_{0}+1}(t)
$$


Solving this differential inequality and noting definition (2.65) of $\delta_{2}$, we obtain

$$
\begin{aligned}
V(t) & \leq\left[(V(0))^{-\sigma_{0}}+\frac{\sigma_{0} \delta_{2} t}{2\left[1+\delta_{2} C_{1} E^{\sigma_{0}}(0)\right]^{\sigma_{0}+1}}\right]^{-1 / \sigma_{0}} \\
& \leq\left[\left(E(0)+\delta_{2} C_{1} E^{\sigma_{0}+1}(0)\right)^{-\sigma_{0}}+\frac{\sigma_{0} \delta_{2} t}{2\left[1+\delta_{2} C_{1} E^{\sigma_{0}}(0)\right]^{\sigma_{0}+1}}\right]^{-1 / \sigma_{0}} \\
& \leq 2 E(0)\left[1+\frac{\sigma_{0} \delta_{2} t}{2(2 E(0))^{-\sigma_{0}}\left[1+\delta_{2} C_{1} E^{\sigma_{0}}(0)\right]^{\sigma_{0}+1}}\right]^{-1 / \sigma_{0}}
\end{aligned}
$$

which, combined with (4.5), implies (2.53).

Finally, if $\left(u^{0}, u^{1}, \theta^{0}\right) \in \mathcal{H}$, in view of (3.84), we can show that Theorem 2.2 still holds by a density argument.

Proof of Theorem 2.3. We first note that $C=C_{0}$ and the constant $C_{1}^{\prime \prime}$ in Lemma 4.1 and the constants $C_{2}^{\prime \prime}$ and $C_{3}^{\prime \prime}$ in Lemma 4.2 become $C_{1}^{\prime}, C_{2}^{\prime}, C_{3}^{\prime}$ in Theorem 2.3, respectively. In this case, $(4.34)$ becomes

$$
\begin{aligned}
V^{\prime}(t) \leq & -\delta E^{\sigma+1}(t)+\left[\delta E^{\sigma}(t)\left(\frac{\sigma \alpha C_{1}^{\prime}}{\beta}+C_{2}^{\prime}\right)-\frac{\alpha}{\beta}\right]\|\nabla \theta(t)\|^{2} \\
& -\left[1-\delta C_{1}^{\prime} \sigma E^{\sigma}(t)\right] \int_{\Gamma_{2}} m \cdot \nu g\left(u^{\prime}\right) \cdot u^{\prime} d \Gamma \\
& +\delta E^{\sigma}(t) \int_{\Gamma_{2}}\left[\left(m \cdot \nu+\frac{C_{0}^{2} k_{1}^{2}}{4 \varepsilon}\right)\left|u^{\prime}\right|^{2}+C_{3}^{\prime} m \cdot \nu\left|g\left(u^{\prime}\right)\right|^{2}\right] d \Gamma .
\end{aligned}
$$

Because the additional term $\frac{C_{0}^{2} k_{1}^{2}}{4 \varepsilon}$ does not contain the factor $m \cdot \nu$, we have to impose condition (2.69) on $\Gamma_{2}$ so that the sum of the integrals on $\Gamma_{2}$ becomes negative if $\delta$ is sufficiently small. The rest of the proof is the same as that of Theorem 2.2 except that $C_{4}$ is replaced by $C_{4}^{\prime}$ and the integral $\int_{\Gamma_{2}} m \cdot \nu g\left(u^{\prime}\right) \cdot u^{\prime} d \Gamma$ is enlarged to $R_{0} \int_{\Gamma_{2}} g\left(u^{\prime}\right) \cdot u^{\prime} d \Gamma$ or reduced to $\eta \int_{\Gamma_{2}} g\left(u^{\prime}\right) \cdot u^{\prime} d \Gamma$.

Proof of Theorem 2.5. The proof of the first part of Theorem 2.5 is the same as that of Theorems 2.2 and 2.3 except that all $u^{\prime}$ and $g\left(u^{\prime}\right)$ are replaced by $u_{i}^{\prime}$ and $h\left(u_{i}^{\prime}\right)$, respectively. Also, in this case, the constant $b$ in (4.40) is taken as

$$
b=\left(\frac{p+1}{2 C_{4} n(p+1-2 q)}\right)^{(p+1-2 q) /(p+1)}
$$

and (4.41) becomes

$$
\begin{aligned}
E^{\sigma}(t) & \int_{\Gamma_{2} \cap\left[\left|u_{i}^{\prime}\right| \leq 1\right]} m \cdot \nu\left[\left|u_{i}^{\prime}\right|^{2}+C_{3}\left|h\left(u_{i}^{\prime}\right)\right|^{2}\right] d \Gamma \\
\leq & \frac{1}{2 n} E^{\sigma(p+1) /(p+1-2 q)} \\
& +\frac{q\left(2 C_{4}\right)^{(p+1) /(2 q)}\left[n R_{0} \operatorname{mes}\left(\Gamma_{2}\right)(p+1-2 q)\right]^{(p+1-2 q) /(2 q)}}{k_{3}(p+1)^{(p+1) /(2 q)}} \\
& \times \int_{\Gamma_{2} \cap\left[\left|u_{i}^{\prime}\right| \leq 1\right]} m \cdot \nu h\left(u_{i}^{\prime}\right) u_{i}^{\prime} d \Gamma .
\end{aligned}
$$

Note that the space dimension $n$ appears here. 
However, the proof of the decay rate $(2.101)$ is a bit different. Hence we present it as follows.

We first look at the case corresponding to Theorem 2.2. Since $p>1$ and $q=1 / p$, as in Remark 2.12, we have

$$
|s|^{2}+C_{3}|h(s)|^{2} \leq C_{5}(h(s) s)^{2 /(p+1)}, \quad|s| \leq 1
$$

where

$$
C_{5}=\frac{1+C_{3} k_{2} k_{3}}{k_{3}} .
$$

It therefore follows that for any $b>0$

$$
\begin{aligned}
E^{\sigma}(t) & \int_{\Gamma_{2} \cap\left[\left|u_{i}^{\prime}\right| \leq 1\right]} m \cdot \nu\left[\left|u_{i}^{\prime}\right|^{2}+C_{3}\left|h\left(u_{i}^{\prime}\right)\right|^{2}\right] d \Gamma \\
\leq & C_{5} \frac{p-1}{p+1} b^{(p+1) /(p-1)} E^{\sigma(p+1) /(p-1)} \\
& +\frac{2 C_{5}}{(p+1) b^{(p+1) / 2}}\left(\int_{\Gamma_{2} \cap\left[\left|u_{i}^{\prime}\right| \leq 1\right]} m \cdot \nu\left(u_{i}^{\prime} h\left(u_{i}^{\prime}\right)\right)^{2 /(p+1)} \Gamma\right)^{(p+1) / 2} \\
\leq & C_{5} \frac{p-1}{p+1} b^{(p+1) /(p-1)} E^{\sigma(p+1) /(p-1)} \\
& +\frac{2 C_{5}}{(p+1) b^{(p+1) / 2}}\left(\int_{\Gamma_{2} \cap\left[\left|u_{i}^{\prime}\right| \leq 1\right]} m \cdot \nu d \Gamma\right)^{(p-1) / 2} \\
\leq & C_{5} \frac{p-1}{p+1} b^{(p+1) /(p-1)} E^{\sigma(p+1) /(p-1)} \\
& +\frac{2 C_{5}\left[R_{0} \operatorname{mes}\left(\Gamma_{2}\right)\right]^{(p-1) / 2}}{(p+1) b^{(p+1) / 2}} \int_{\Gamma_{2} \cap\left[\left|u_{i}^{\prime}\right| \leq 1\right]} m \cdot \nu u_{i}^{\prime} h\left(u_{i}^{\prime}\right) d \Gamma
\end{aligned}
$$

Thus, by taking

$$
b=\left(\frac{p+1}{2 C_{5}(p-1)}\right)^{(p-1) /(p+1)}
$$

we obtain

$$
\begin{gathered}
E^{\sigma}(t) \int_{\Gamma_{2} \cap\left[\left|u_{i}^{\prime}\right| \leq 1\right]} m \cdot \nu\left[\left|u_{i}^{\prime}\right|^{2}+C_{3}\left|h\left(u_{i}^{\prime}\right)\right|^{2}\right] d \Gamma \\
\leq \frac{1}{2} E^{\sigma(p+1) /(p-1)}+\frac{\left(2 C_{5}\right)^{(p+1) / 2}\left[R_{0} \operatorname{mes}\left(\Gamma_{2}\right)(p-1)\right]^{(p-1) / 2}}{(p+1)^{(p+1) / 2}} \\
\quad \times \int_{\Gamma_{2} \cap\left[\left|u_{i}^{\prime}\right| \leq 1\right]} m \cdot \nu u_{i}^{\prime} h\left(u_{i}^{\prime}\right) d \Gamma .
\end{gathered}
$$


It therefore follows from (4.34) that

$$
\begin{aligned}
& V^{\prime}(t) \leq-\delta E^{\sigma+1}(t)+\left[\delta E^{\sigma}(t)\left(\frac{\sigma \alpha C_{1}}{\beta}+C_{2}\right)-\frac{\alpha}{\beta}\right]\|\nabla \theta(t)\|^{2} \\
& +\left[\delta C_{1} \sigma E^{\sigma}(0)+\frac{\delta C_{4} E^{\sigma}(0)}{k_{3}}-1\right] \int_{\Gamma_{2} \cap\left[\left|u_{i}^{\prime}\right| \geq 1\right]} m \cdot \nu u_{i}^{\prime} h\left(u_{i}^{\prime}\right) d \Gamma \\
& +\left[\delta C_{1} \sigma E^{\sigma}(0)-1\right] \int_{\Gamma_{2} \cap\left[\left|u_{i}^{\prime}\right| \leq 1\right]} m \cdot \nu u_{i}^{\prime} h\left(u_{i}^{\prime}\right) d \Gamma \\
& +\delta E^{\sigma}(t) \int_{\Gamma_{2} \cap\left[\left|u_{i}^{\prime}\right| \leq 1\right]} m \cdot \nu\left[\left|u_{i}^{\prime}\right|^{2}+C_{3}\left|h\left(u_{i}^{\prime}\right)\right|^{2}\right] d \Gamma \\
& \leq-\delta E^{\sigma+1}(t)+\frac{\delta}{2} E^{\sigma(p+1) /(p-1)} \\
& +\left[\delta E^{\sigma}(t)\left(\frac{\sigma \alpha C_{1}}{\beta}+C_{2}\right)-\frac{\alpha}{\beta}\right]\|\nabla \theta(t)\|^{2} \\
& +\left[\delta C_{1} \sigma E^{\sigma}(0)+\frac{\delta C_{4} E^{\sigma}(0)}{k_{3}}-1\right] \int_{\Gamma_{2} \cap\left[\left|u_{i}^{\prime}\right| \geq 1\right]} m \cdot \nu u_{i}^{\prime} h\left(u_{i}^{\prime}\right) d \Gamma \\
& +\left[\delta C_{1} \sigma E^{\sigma}(0)-1\right] \int_{\Gamma_{2} \cap\left[\left|u_{i}^{\prime}\right| \leq 1\right]} m \cdot \nu u_{i}^{\prime} h\left(u_{i}^{\prime}\right) d \Gamma \\
& +\delta \frac{\left(2 C_{5}\right)^{(p+1) / 2}\left[R_{0} \operatorname{mes}\left(\Gamma_{2}\right)(p-1)\right]^{(p-1) / 2}}{(p+1)^{(p+1) / 2}} \\
& \times \int_{\Gamma_{2} \cap\left[\left|u_{i}^{\prime}\right| \leq 1\right]} m \cdot \nu u_{i}^{\prime} h\left(u_{i}^{\prime}\right) d \Gamma \text {. }
\end{aligned}
$$

By taking $\delta=\delta_{2}($ see $(2.65))$ and $\sigma=(p-1) / 2$, we obtain

$$
V^{\prime}(t) \leq-\frac{\delta_{2}}{2} E^{(p+1) / 2}(t)
$$

The remainder of the proof is the same as that of Theorem 2.2.

The proof of the case corresponding to Theorem 2.3 is similar.

We now use the method developed in [41] to prove Theorem 2.7.

Proof of Theorem 2.7. If $E\left(t_{0}\right)=0$ for some $t_{0} \geq 0$, then, by $(2.42)$, we have $E(t) \equiv 0$ for $t \geq t_{0}$ and then the theorem holds. Therefore, we may assume that $E(t)>0$ for $t \geq 0$. This assumption ensures that, in the following proof, $\varphi^{\prime \prime}(a E(t))$ makes sense since we have assumed that $\varphi(s)$ is twice differentiable outside $s=0$.

We first note that $C=C_{0}$ and the constant $C_{1}^{\prime \prime}$ in Lemma 4.1 and the constants $C_{2}^{\prime \prime}$ and $C_{3}^{\prime \prime}$ in Lemma 4.2 become $C_{1}^{\prime}, C_{2}^{\prime}, C_{3}^{\prime}$ in Theorem 2.3 , respectively. Set

$$
V(t)=E(t)+\delta \varphi^{\prime}(a E(t)) F(t),
$$


where the constant $a$ is given by (2.116). By (2.42), (4.10) and Lemmas 4.1 and 4.2 , we have

$$
\begin{aligned}
& V^{\prime}(t)=E^{\prime}(t)+\delta a \varphi^{\prime \prime}(a E(t)) E^{\prime}(t) F(t)+\delta \varphi^{\prime}\left(a E(t) F^{\prime}(t)\right. \\
& \leq\left[1-\delta a C_{1}^{\prime} \varphi^{\prime \prime}(a E(0)) E(0)\right] E^{\prime}(t)-\delta \varphi^{\prime}(a E(t)) E(t) \\
& +\delta C_{2}^{\prime} \varphi^{\prime}(a E(0))\|\nabla \theta(t)\|^{2} \\
& +\delta \varphi^{\prime}(a E(t)) \int_{\Gamma_{2}}\left[\left(m \cdot \nu+2 C_{0}^{2} k_{1}^{2}\left|u^{\prime}\right|^{2}+C_{3}^{\prime} m \cdot \nu \sum_{i=1}^{n}\left|h\left(u_{i}^{\prime}\right)\right|^{2}\right] d \Gamma\right. \\
& \leq-\delta \varphi^{\prime}(a E(t)) E(t)+\left[\delta C_{2}^{\prime} \varphi^{\prime}(a E(0))+\alpha \delta a C_{1}^{\prime} \varphi^{\prime \prime}(a E(0)) E(0) / \beta-\alpha / \beta\right]\|\nabla \theta(t)\|^{2} \\
& +\left[\eta \delta a C_{1}^{\prime} \varphi^{\prime \prime}(a E(0)) E(0)+\delta \varphi^{\prime}(a E(0))\left[R_{0}+2 C_{0}^{2} k_{1}^{2}+C_{3}^{\prime} k_{2}^{2} R_{0}\right] / k_{3}-\eta\right] \\
& \times \sum_{i=1}^{n} \int_{\Gamma_{2} \cap\left[\left|u_{i}^{\prime}\right| \geq\right]} h\left(u_{i}^{\prime}\right) u_{i}^{\prime} d \Gamma \\
& +\left[\eta \delta a C_{1}^{\prime} \varphi^{\prime \prime}(a E(0)) E(0)-\eta\right] \sum_{i=1}^{n} \int_{\Gamma_{2} \cap\left[\left|u_{i}^{\prime}\right| \leq 1\right]} h\left(u_{i}^{\prime}\right) u_{i}^{\prime} d \Gamma \\
& \text { (note that }\left|u^{\prime}\right|^{2} \leq\left|u^{\prime}\right|^{2 q} \text { since } q \leq 1 \text { and }\left|u_{i}^{\prime}\right| \leq 1 \text { ) } \\
& +\delta\left[R_{0}+2 C_{0}^{2} k_{1}^{2}+C_{3}^{\prime} k_{2}^{2} R_{0}\right] \varphi^{\prime}(a E(t)) \sum_{i=1}^{n} \int_{\Gamma_{2} \cap\left[\left|u_{i}^{\prime}\right| \leq 1\right]}\left|u_{i}^{\prime}\right|^{2 q} d \Gamma \\
& \leq-\delta \varphi^{\prime}(a E(t)) E(t) \\
& +\left[\eta \delta a C_{1}^{\prime} \varphi^{\prime \prime}(a E(0)) E(0)-\eta\right] \sum_{i=1}^{n} \int_{\Gamma_{2} \cap\left[\left|u_{i}^{\prime}\right| \leq 1\right]} h\left(u_{i}^{\prime}\right) u_{i}^{\prime} d \Gamma \\
& +\delta\left[R_{0}+2 C_{0}^{2} k_{1}^{2}+C_{3}^{\prime} k_{2}^{2} R_{0}\right] \varphi^{\prime}(a E(t)) \sum_{i=1}^{n} \int_{\Gamma_{2} \cap\left[\left|u_{i}^{\prime}\right| \leq 1\right]}\left|u_{i}^{\prime}\right|^{2 q} d \Gamma,
\end{aligned}
$$

if $\delta$ is small enough. Let $\varphi^{*}$ denote the dual of $\varphi$ in the sense of Young (for definition, see [4, p. 64]). Then, by Young's inequality [4, p. 64] and Jensen's inequality [49], we deduce

$$
\begin{aligned}
& \varphi^{\prime}(a E(t)) \int_{\Gamma_{2} \cap\left[\left|u_{i}^{\prime}\right| \leq 1\right]}\left|u_{i}^{\prime}\right|^{2 q} d \Gamma \\
& \quad=\operatorname{mes}\left(\Gamma_{2} \cap\left[\left|u_{i}^{\prime}\right| \leq 1\right]\right) \varphi^{\prime}(a E) \frac{1}{\operatorname{mes}\left(\Gamma_{2} \cap\left[\left|u_{i}^{\prime}\right| \leq 1\right]\right)} \int_{\Gamma_{2} \cap\left[\left|u_{i}^{\prime}\right| \leq 1\right]}\left|u_{i}^{\prime}\right|^{2 q} d \Gamma \\
& \quad \leq \operatorname{mes}\left(\Gamma_{2} \cap\left[\left|u_{i}^{\prime}\right| \leq 1\right]\right)\left[\varphi^{*}\left(\varphi^{\prime}(a E)\right)+\varphi\left(\frac{1}{\operatorname{mes}\left(\Gamma_{2} \cap\left[\left|u_{i}^{\prime}\right| \leq 1\right]\right)} \int_{\Gamma_{2} \cap\left[\left|u_{i}^{\prime}\right| \leq 1\right]}\left|u_{i}^{\prime}\right|^{2 q} d \Gamma\right)\right] \\
& \leq \operatorname{mes}\left(\Gamma_{2}\right) \varphi^{*}\left(\varphi^{\prime}(a E)\right)+\int_{\Gamma_{2} \cap\left[\left|u_{i}^{\prime}\right| \leq 1\right]} \varphi\left(\left|u_{i}^{\prime}\right|^{2 q}\right) d \Gamma \\
& \leq \operatorname{mes}\left(\Gamma_{2}\right) \varphi^{*}\left(\varphi^{\prime}(a E)\right)+\int_{\Gamma_{2} \cap\left[\left|u_{i}^{\prime}\right| \leq 1\right]} u_{i}^{\prime} h\left(u_{i}^{\prime}\right) d x .
\end{aligned}
$$


Hence, if $\delta$ is small enough, then it follows from (4.60) and (4.61) that

$$
V^{\prime}(t) \leq-\delta \varphi^{\prime}(a E(t)) E(t)+n \delta\left[R_{0}+2 C_{0}^{2} k_{1}^{2}+C_{3}^{\prime} k_{2}^{2} R_{0}\right] \operatorname{mes}\left(\Gamma_{2}\right) \varphi^{*}\left(\varphi^{\prime}(a E)\right) .
$$

By the definition of the dual $\varphi^{*}(t)$ of $\varphi(s), \varphi^{*}(t)$ is the Legendre transform of $\varphi(s)$, which is given by (see $[4$, pp. 61-62])

$$
\varphi^{*}(t)=t \varphi^{\prime-1}(t)-\varphi\left[\varphi^{\prime-1}(t)\right] .
$$

It therefore follows from (4.62) that

$$
\begin{aligned}
V^{\prime}(t) \leq & -\delta \varphi^{\prime}(a E(t)) E(t) \\
& +n \delta\left[R_{0}+2 C_{0}^{2} k_{1}^{2}+C_{3}^{\prime} k_{2}^{2} R_{0}\right] \operatorname{mes}\left(\Gamma_{2}\right)\left[a \varphi^{\prime}(a E(t)) E(t)-\varphi(a E(t))\right] \\
= & -n \delta\left[R_{0}+2 C_{0}^{2} k_{1}^{2}+C_{3}^{\prime} k_{2}^{2} R_{0}\right] \operatorname{mes}\left(\Gamma_{2}\right) \varphi(a E(t)) \\
& -\frac{\delta}{2} \varphi^{\prime}(a E(t)) E(t)
\end{aligned}
$$

with

$$
a=\frac{1}{2 n\left[R_{0}+2 C_{0}^{2} k_{1}^{2}+C_{3}^{\prime} k_{2}^{2} R_{0}\right] \operatorname{mes}\left(\Gamma_{2}\right)} .
$$

On the other hand, since $\varphi(s)$ and $\varphi^{\prime}(s)$ are positive and increasing on $[0, \infty)$, it follows from (4.10) that

$$
\left[1-\delta C_{1}^{\prime} \varphi^{\prime}(a E(0))\right] E(t) \leq V(t) \leq\left[1+\delta C_{1}^{\prime} \varphi^{\prime}(a E(0))\right] E(t) .
$$

It therefore follows from (4.64) that

$$
\begin{aligned}
V^{\prime}(t) \leq & -\frac{\delta V(t)}{2\left(1+\delta C_{1}^{\prime} \varphi^{\prime}(a E(0))\right)} \varphi^{\prime}\left(\frac{a V(t)}{1+\delta C_{1}^{\prime} \varphi^{\prime}(a E(0))}\right) \\
& -n \delta\left[R_{0}+2 C_{0}^{2} k_{1}^{2}+C_{3}^{\prime} k_{2}^{2} R_{0}\right] \operatorname{mes}\left(\Gamma_{2}\right) \varphi\left(\frac{a V(t)}{1+\delta C_{1}^{\prime} \varphi^{\prime}(a E(0))}\right) .
\end{aligned}
$$

This is (2.115).

It remains to prove (2.118). We argue by contradiction. Suppose that $E(t)$ does not tend to zero as $t \rightarrow \infty$. Since $E(t)$ is decreasing on $[0, \infty)$, we have

$$
E(t) \geq \sigma>0, \quad \forall t \geq 0
$$

and by (4.66), we have

$$
V(t) \geq \beta>0, \quad \forall t \geq 0
$$

Thus we have

$$
\varphi^{\prime}\left(\frac{a V(t)}{b}\right) \geq \gamma>0, \quad \forall t \geq 0 .
$$

It therefore follows from (2.115) that

$$
V^{\prime}(t) \leq-\frac{\delta \gamma}{2 b} V(t), \quad \forall t \geq 0
$$

which is in contradiction with (4.69). This completes the proof. 
Proof of Corollary 2.9. By Theorem 2.7, it suffices to prove that, for $t$ large enough, (2.115) becomes $(2.121)$. Since $h$ is odd in $[-1,1]$ and $s^{1 /(2 q)} h\left(s^{1 /(2 q)}\right)$ is convex in $[0,1]$. we can take for $0 \leq s \leq 1$

$$
\varphi(s)=s^{1 /(2 q)} h\left(s^{1 /(2 q)}\right)
$$

and then

$$
\varphi^{\prime}(s)=\frac{1}{2 q}\left[s^{(1-2 q) /(2 q)} h\left(s^{1 /(2 q)}\right)+s^{(1-q) / q} h^{\prime}\left(s^{1 /(2 q)}\right)\right] .
$$

On the other hand, by $(2.118)$, there exists $T>0$ such that

$$
V(t) \leq 1, \quad \forall t \geq T .
$$

Thus, for $t \geq T$, substituting (4.72) and (4.73) into (2.115), we obtain (2.121).

Proof of Corollary 2.10. If $h$ satisfies (2.95), then we can take

$$
\varphi(s)=k_{3} s^{(p+1) /(2 q)}, \quad \text { for } s \geq 0 .
$$

Thus, (2.115) becomes

$$
V^{\prime}(t)=-K(V(t))^{(p+1) /(2 q)},
$$

where $K$ is a positive constant independent of $V$. Hence, the decay properties (2.52) and (2.53) follow from (4.76).

5. Further comments. In the proof of Theorems 2.2-2.7, inequality (2.25) of Poincaré type plays a key role. This inequality is guaranteed by assumption (2.16). If this assumption does not hold, i.e., $\Gamma_{1}=\emptyset$ and $a(x) \equiv 0$, then this inequality is no longer true for all $u \in H^{1}(\Omega)$. When $\Gamma_{1}=\phi$ and $a(x) \equiv 0$, in order to guarantee the coercivity of the energy, it is natural to look for a closed subspace or subset $\mathcal{W}$ of $\left.\left(H^{1}(\Omega)\right)^{n} \times\left(L^{2} \Omega\right)\right)^{n} \times$ $L^{2}(\Omega)$ invariant under the flow generated by the semigroup such that the energy norm (2.20) on $\mathcal{W}$ is equivalent to the usual one induced by $\left(H^{1}(\Omega)\right)^{n} \times\left(L^{2}(\Omega)\right)^{n} \times L^{2}(\Omega)$.

Naturally, one is tempted to consider the following space of functions with zero average:

$$
\begin{array}{r}
\mathcal{H}_{0}=\left\{(u, v, \theta) \in\left(H^{1}(\Omega)\right)^{n} \times\left(L^{2}(\Omega)\right)^{n} \times L^{2}(\Omega):\right. \\
\left.\int_{\Omega} u(x) d x=\int_{\Omega} v(x) d x=0\right\},
\end{array}
$$

on which the energy norm is really equivalent to the usual one. Unfortunately, $\mathcal{H}_{0}$ is not invariant. To see this, we define the function

$$
f(t)=\int_{\Omega} u(t) d x
$$

We look at the special case where $g(u)=u$. Take sufficiently regular initial $\left(u^{0}, u^{1}, \theta^{0}\right) \in$ $\mathcal{H}_{0}$ such that

$$
\int_{\Gamma} m \cdot \nu u^{1} d \Gamma \neq 0
$$


and let $u, \theta$ be the solution of (2.7) corresponding to this initial data. By the continuity of $u^{\prime}(t)$ with respect to $t$, we have that

$$
\int_{\Gamma} m \cdot \nu u^{\prime}(t) d \Gamma \neq 0
$$

for $t>0$ small enough. It therefore follows that

$$
\begin{aligned}
f^{\prime \prime}(t) & =\int_{\Omega} u^{\prime \prime}(t) d x \\
& =\int_{\Omega}[\mu \Delta u+(\lambda+\mu) \nabla \operatorname{div} u-\alpha \nabla \theta] d x \\
& =\int_{\Gamma}\left[\mu \frac{\partial u}{\partial \nu}+(\lambda+\mu) \operatorname{div}(u) \nu-\alpha \theta \nu\right] d \Gamma \\
& =-\int_{\Gamma} m \cdot \nu u^{\prime}(t) d \Gamma \\
& \neq \equiv .
\end{aligned}
$$

Hence, $\int_{\Omega} u(t) d x$ and $\int_{\Omega} u^{\prime}(t) d x$ are not always equal to zero along the solution trajectories of (2.7).

On the other hand, we do have the following conserved quantity:

$$
\int_{\Omega} u^{\prime}(t) d x+\int_{0}^{t} \int_{\Gamma} m \cdot \nu g\left(u^{\prime}(t)\right) d \Gamma d t \equiv C \text { (a constant) }
$$

since, by (2.7), we have

$$
\int_{\Omega} u^{\prime \prime}(t) d x+\int_{\Gamma} m \cdot \nu g\left(u^{\prime}(t)\right) d \Gamma \equiv 0
$$

If $g(u)$ is linear, i.e., $g(u)=k u$, then we can easily find an invariant subspace $\mathcal{W}$ as follows:

$$
\mathcal{W}=V \times L^{2}(\Omega)
$$

where

$$
V=\left\{(u, v) \in\left(H^{1}(\Omega)\right)^{n} \times\left(L^{2}(\Omega)\right)^{n}: \int_{\Gamma} k m \cdot \nu u d \Gamma+\int_{\Omega} v d x=0\right\}
$$

Moreover, the energy norm on $\mathcal{W}$ is equivalent to the usual one. However, for the general nonlinear boundary feedbacks, it is difficult to find such an invariant closed subset. Thus, the case that $\Gamma_{1}=\emptyset$ and $a(x) \equiv 0$ with $g$ nonlinear is open.

Acknowledgment. Part of this work was done when the first author visited the Departamento de Matemática Aplicada, Universidad Complutense de Madrid, in May, 1998. He thanks their hospitality and financial support. 


\section{REFERENCES}

[1] R. Adams, Sobolev Spaces, Academic Press, New York, 1975

[2] S. Agmon, Lectures on Elliptic Boundary Value Problems, D. Van Nostrand Company, Inc., Princeton, NJ, 1965

[3] F. Alabau and V. Komornik, Boundary observability, controllability and stabilization of linear elastodynamic systems, SIAM J. Control Optim. 37, no. 2, 521-542 (1999)

[4] V. I. Arnold, Mathematical Methods of Classical Mechanics, Springer-Verlag, New York, 1989

[5] G. Avalos and I. Lasiecka, Exponential stability of a thermoelastic system with free boundary conditions without mechanical dissipation, SIAM J. Math. Anal. 29, 155-182 (1998)

[6] V. Barbu, Nonlinear Semigroups and Differential Equations in Banach Spaces, Noordhoff, Leyden, The Netherlands, 1976

[7] E. Bisognin, V. Bisognin, G. P. Menzala, and E. Zuazua, On exponential stability for the Von Kármán equations in the presence of thermal effects, Math. Methods Appl. Sci. 21, 393-416 (1998)

[8] H. Brezis, Analyse Fonctionnelle: Théorie et Applications, Masson, Paris, 1983

[9] J. A. Burns, Z. Y. Liu, and S. Zheng, On the energy decay of a linear thermoelastic bar, J. Math. Anal. Appl. 179, 574-591 (1993)

[10] G. Chen, Energy decay estimates and exact boundary value controllability for the wave equation in a bounded domain, J. Math. Pures Appl. 58, 249-273 (1979)

[11] F. Conrad and B. Rao, Decay of solutions of the wave equation in a star-shaped domain with nonlinear boundary feedback, Asymptotic Anal. 7, 159-177 (1993)

[12] C. M. Dafermos, On the existence and the asymptotic stability of solutions to the equations of linear thermoelasticity, Arch. Rational Mech. Anal. 29, 241-271 (1968)

[13] R. Dautray and J. L. Lions, Mathematical Analysis and Numerical Methods for Science and Technology, Vol. 2, Functional and Variational Methods, Springer-Verlag, Berlin, 1992

[14] P. Grisvard, Singularities in Boundary Value Problems, Masson, Paris, 1992

[15] P. Grisvard, Elliptic Problems in Nonsmooth Domains, Pitman, London, 1985

[16] P. Grisvard, Contrôlabilité exacte des solutions de l'équation des ondes en présence de singularités, J. Math. Pures Appl. 68, 215-259 (1989)

[17] S. W. Hansen, Exponential energy decay in a linear thermoelastic rod, J. Math. Anal. Appl. 167, 429-442 (1992)

[18] M. A. Horn, Implications of sharp trace regularity results on boundary stabilization of the system of linear elasticity, J. Math. Anal. Appl. 223, 126-150 (1998)

[19] M. A. Horn, Sharp trace regularity for the solutions of the equations of dynamic elasticity, J. Math. Systems Estim. Control 8 (2), 217-219 (1998)

[20] J. U. Kim, On the energy decay of a linear thermoelastic bar and plate, SIAM J. Math. Anal. 23, 889-899 (1992)

[21] V. Komornik, Exact Controllability and Stabilization: The Multiplier Method, John Wiley and Sons, Masson, Paris, 1994

[22] V. Komornik and B. Rao, Boundary stabilization of compactly coupled wave equations, Asymptotic Anal. 14, 339-359 (1997)

[23] V. Komornik and E. Zuazua, A direct method for the boundary stabilization of the wave equation, J. Math. Pures Appl. 69, 33-54 (1990)

[24] A. D. Kovalenko, Thermoelasticity, Basic Theory and Applications, Wolters-Noordhoff Publishing, Gröningen, Netherlands, 1969

[25] J. Lagnese, Decay of solutions of wave equations in a bounded region with boundary dissipation, J. Differential Equations 50, 163-182 (1983)

[26] J. Lagnese, Boundary stabilization of linear elastodynamic systems, SIAM J. Control Optim. 21, 968-984 (1983)

[27] J. Lagnese, Boundary Stabilization of Thin Plates, SIAM Studies in Applied Mathematics, vol. 10, SIAM Publications, Philadelphia, 1989

[28] J. Lagnese, Uniform asymptotic energy estimates for solutions of the equations of dynamic plane elasticity with nonlinear dissipation at the boundary, Nonlinear Anal. 16, 35-54 (1991)

[29] J. Lagnese and G. Leugering, Uniform stabilization of a nonlinear beam by nonlinear boundary feedback, J. Differential Equations 91, 355-388 (1991)

[30] J. Lagnese and J. L. Lions, Modelling Analysis and Control of Thin Plates, Masson, Paris, 1989 
[31] I. Lasiecka, Stabilization of wave and plate-like equations with nonlinear dissipation on the boundary, J. Differential Equations 79, 340-381 (1989)

[32] I. Lasiecka and D. Tataru, Uniform boundary stabilization of semilinear wave equations with nonlinear boundary damping, Differential Integral Equations 6, 507-533 (1993)

[33] G. Lebeau and E. Zuazua, Sur la décroissance non uniforme de l'énérgie dans le système de la thermoélasticité linéaire, C. R. Acad. Sci. Paris Sér. I. Math. 324, 409-415 (1997)

[34] G. Leugering, On boundary feedback stabilization of a viscoelastic membrane, Dynam. Stability Systems 4, 71-79 (1989)

[35] G. Leugering, On boundary feedback stabilization of a viscoelastic beam, Proc. Roy. Soc. Edinburgh, Sect. A 114, 57-69 (1990)

[36] J. L. Lions and E. Magenes, Non-homogeneous Boundary Value Problems and Applications, vols. $I$ and II, Springer-Verlag, New York, 1972

[37] W. J. Liu, Partial exact controllability and exponential stability of the higher-dimensional linear thermoelasticity, ESAIM Contrôle Optim. Calc. Var. 3, 23-48 (1998)

[38] W. J. Liu, Exact Controllability for Some Partial Differential Equations of Evolutional Type, Ph.D. Thesis, University of Wollongong, Australia, 1997

[39] W. J. Liu, The exponential stabilization of the higher-dimensional linear thermoviscoelasticity, J. Math. Pures Appl. 77, 355-386 (1998)

[40] Z. Liu and S. Zheng, Exponential stability of the semigroup associated with a thermoelastic system, Quart. Appl. Math. LI, 535-545 (1993)

[41] W. J. Liu and E. Zuazua, Decay rates for dissipative wave equations, Ricerche de Matematica 48, 61-75 (1999)

[42] G. P. Menzala and E. Zuazua, Explicit exponential decay rates for solutions of von Kármán's system of thermoelastic plates, C. R. Acad. Sci Paris Sér. I Math. 324, 49-54 (1997)

[43] G. P. Menzala and E. Zuazua, Energy decay rates for the Von Kármán system of thermoelastic plates, Differential Integral Equations, to appear.

[44] K. Narukawa, Boundary value control of thermoelastic systems, Hiroshima Math. J. 13, 227-272 (1983)

[45] L. A. F. Oliveira, Exponential decay in thermoelasticity, Commun. Appl. Anal. 1, 113-118 (1997)

[46] J. E. M. Rivera, Decomposition of the displacement vector field and decay rates in linear thermoelasticity, SIAM J. Math. Anal. 24, 390-406 (1993)

[47] J. E. M. Rivera, Asymptotic behaviour in n-dimensional thermoelasticity, Appl. Math. Lett. 10, 47-53 (1997)

[48] J. E. M. Rivera and M. L. Oliveira, Stability in inhomogeneous and anisotropic thermoelasticity, Boll. Un. Mat. Ital. A A (7) 11, 115-127 (1997)

[49] W. Rudin, Real and Complex Analysis, second edition, McGraw-Hill, Inc., New York, 1974

[50] H. K. Wang and G. Chen, Asymptotic behavior of solutions of the one-dimensional wave equation with a nonlinear boundary stabilizer, SIAM J. Control Optim. 27, 758-775 (1989)

[51] E. Zuazua, Uniform stabilization of the wave equation by nonlinear boundary feedback, SIAM J. Control Optim. (2) 28, 466-477 (1990) 